\title{
26. DATA REPORT: X-RADIOGRAPHY OF SELECTED, PREDOMINANTLY VARVED INTERVALS AT HOLE 893 ${ }^{1}$
}

\author{
Carina B. Lange ${ }^{2}$ and Arndt Schimmelmann ${ }^{3}$
}

\section{INTRODUCTION}

The predominantly varved sediments of the uppermost $3.5 \mathrm{~m}$ of Core 146-893A-1H cover a depth range of Santa Barbara Basin (SBB) sediment that is accessible by Kasten coring and other gravity coring techniques. These sediments previously have been the focus of many paleoceanographic studies (reviewed by Lange et al., in press), This sediment interval gains additional value when it can be stratigraphically matched against other paleoceanographic records from nearby sites. This study provides a preliminary framework for later, more detailed correlation among other SBB sites.

High-resolution paleoclimatic reconstruction based on varve records requires accurate age-assignment of varves (e.g., Baumgartner et al., 1991). Annual resolution over at least the past 1,000 years permits cross-correlation and detailed comparisons with other available paleoclimatic time series, with comparably high resolution provided by ice cores, tree rings, coral bands, and other varve series (Baumgartner et al., 1989; Lange et al., in press). X-radiography of sediment slabs is an efficient way to document the sedimentological aspect and structure variation of varved sediment (Soutar et al., 1982). Most of the SBB varve record of this millennium has already been described in detail (Soutar and Crill, 1977; Schimmelmann et al., 1990, 1992; Lange et al., in press). In addition, older sediments reaching back over the past 2,000 years have been X-radiographed by R. Byrne et al. (unpubl. data), T. Baumgartner, and A. Soutar (pers. comm., 1993). Furthermore, in August of 1993 we retrieved from the central SBB fresh sediment material, which extends to approximately 200 A.D.

The main objective of this study is to provide unambiguous anchor dates for the topmost sediment of Hole 893A. This is accomplished by illustrating cross-correlations between X-radiographs from selected intervals of Hole 893A with X-radiographic records from earlier core material. We also provide a detailed description of the varve stratigraphy, including clarity of the boundaries on the Xradiographs and the presence of nonlaminated intervals interrupting the varve sequences.

\section{MATERIALS AND METHODS}

We obtained 32 sediment slabs from selected intervals of the upper $24 \mathrm{~m}$ of Hole 893A (Section 146-893-1H-1 through 3H-6; Table 1). This upper sequence (Subunit IA; Shore-based Scientific Party, 1994) contains mainly olive gray (5Y 4/2) diatom nannofossil clayey silt and diatom nannofossil silty clay. It is characterized by the pres-

'Kennett, J.P., Baldauf, J.G., and Lyle, M. (Eds.), 1995. Proc. ODP, Sci. Results, 146 (Pt. 2): College Station, TX (Ocean Drilling Program).

2Scripps Institution of Oceanography, Geological Research Division 0215, La Jolla, CA 92093-0215, U.S.A.

'Department of Geological Sciences, Biogeochemical Laboratories, Geology 317 , Indiana University, Bloomington, IN 47405-5101, U.S.A ence of variably preserved laminations throughout, intercalated with thin horizons of homogenous sediment. A prominent, notably thicker, nonlaminated interval extends from 17.5 to $20.5 \mathrm{mbsf}$.

\section{Slabbing Procedure}

The slabbing procedure followed the technique described by Schimmelmann et al. (1990), which uses anodic charging to produce a lubricating layer of hydrogen gas on a metallic slabbing device to assist in slicing the sediment. Intervals for slabbing were chosen after the surface of the working halfcore had been scraped with an anodically charged metal blade to expose sedimentary features such as varves and boundaries with nonlaminated sediment intervals. The slabs measured $15-20 \mathrm{~cm}$ in length, $5 \mathrm{~cm}$ in width, and were cut to a uniform thickness of $1 \mathrm{~cm}$ for optimal results from the X-radiography.

Sediment slabs were wrapped in clear polyethylene film to prevent desiccation. Slab orientation and archive number were marked by attaching a unique symbol (made from bent copper wire) at one end of each slab. The X-radiographic images of the copper wire symbols proved to be reliable identifiers. The slabs were stored in plastic containers in a dark cold room.

\section{X-radiography}

$\mathrm{X}$-radiography of sediment slabs was used for documenting the sediment structure, for varve-counting, and for developing the crosscorrelations with previously collected SBB cores. A modified medical X-ray unit with a 40-kV head at the Scripps Institution of Oceanography was employed for this work. We exposed $8 \times 10$-in. Kodak Industrex M film for 80 to 120 seconds, depending on the overall water content of a particular slab. Each slab was X-radiographed repeatedly at slightly different angles to provide the best resolution of varve boundaries. The ragged longitudinal margin of the X-radiographic images of Hole 893A slabs in Figures 1-3 indicates the area of contact with the core liner of the working halfcore where material had broken loose and adhered to the liner during extraction of the slab. A complete set of X-radiograph contact prints and negatives is kept on file at the Geological Research Division, Scripps Institution of Oceanography.

\section{RESULTS}

A dark band on an X-radiograph contact print indicates strong absorption of X-rays because of the relatively higher density of terrigenous mineral content of a particular sediment layer. Such mineralrich subannual laminae have been identified with winter to early spring deposition (Emery and Hülsemann, 1962; Soutar and Crill, 1977; Reimers et al., 1990); more porous subannual laminae, relatively poor in minerals, correspond to increased summer deposition of biogenous material and appear lighter in the X-radiograph contact print. The dark and light laminae pairs have been shown to represent 
Table 1. Summary of sediment slab samples of Hole 893A, including a general description of the dominant structures.

\begin{tabular}{|c|c|c|c|c|}
\hline $\begin{array}{l}\text { Core, section, } \\
\text { interval }(\mathrm{cm})\end{array}$ & $\begin{array}{l}\text { Depth } \\
\text { (mbsf) }\end{array}$ & $\begin{array}{l}\text { Volume } \\
\left(\mathrm{cm}^{3}\right)\end{array}$ & Slab \# & Comments \\
\hline \multicolumn{5}{|l|}{$146-893 \mathrm{~A}-$} \\
\hline IH-1, 65-80 & 0.65 & 45 & $1^{*}$ & Partially laminated \\
\hline $1 \mathrm{H}-\mathrm{I}, 100-115$ & 1.00 & 45 & $2^{*}$ & Well laminated \\
\hline $\mathrm{IH}-1,115-130$ & 1.15 & 45 & 3 & Partially laminated \\
\hline $1 \mathrm{H}-1,130-150$ & 1.30 & 60 & 4 & Laminated, I gray layer \\
\hline $1 \mathrm{H}-2,0-15$ & 1.50 & 45 & 5 & Laminated, I gray layer \\
\hline $1 \mathrm{H}-2,16-31$ & 1.66 & 45 & 6 & Partially laminated \\
\hline $\mathrm{IH}-2,3 \mathrm{I}-46$ & 1.81 & 45 & $7^{*}$ & Well laminated \\
\hline $\mathrm{IH}-2,46-62$ & 1.96 & 45 & 8 & Laminated, I gray layer \\
\hline $\mathrm{IH}-2,62-77$ & 2.12 & 45 & 9 & Partially laminated \\
\hline $\mathrm{IH}-2,78-93$ & 2.28 & 45 & 10 & Partially laminated \\
\hline $1 \mathrm{H}-2,93-109$ & 2.43 & 48 & II & Weakly laminated \\
\hline IH-2, 109-124 & 2.59 & 45 & $12 *$ & Laminated, 1 gray layer \\
\hline $\mathrm{IH}-2,124-139$ & 2.74 & 45 & 13 & Laminated \\
\hline IH-2, 139-152 & 2.89 & 39 & 14 & Weakly laminated \\
\hline IH-3, 67-82 & 3.69 & 45 & 15 & Well laminated \\
\hline IH-4, 67-82 & 5.19 & 45 & 16 & Partially laminated \\
\hline IH $-5,10-25$ & 6.12 & 45 & 17 & Partially laminated \\
\hline $2 \mathrm{H}-1,26-41$ & 6.76 & 45 & 18 & Partially laminated \\
\hline $2 \mathrm{H}-1,130-145$ & 7.80 & 45 & 19 & Partially laminated \\
\hline $2 \mathrm{H}-2,95-110$ & 8.99 & 45 & $20 *$ & Partially laminated, $w /$ shell \\
\hline $2 \mathrm{H}-3,5-20$ & 9.59 & 45 & 21 & Partially laminated \\
\hline $2 \mathrm{H}-4,1-16$ & 11.06 & 45 & 22 & Partially laminated \\
\hline $2 \mathrm{H}-4,130-150$ & 12.35 & 60 & $23^{*}$ & Partially laminated \\
\hline $2 \mathrm{H}-6,128-143$ & 15.33 & 45 & 24 & Laminated, w/ shell \\
\hline $2 \mathrm{H}-7,1-16$ & 15.56 & 45 & 25 & Partially laminated \\
\hline $2 \mathrm{H}-7,37-52$ & 15.92 & 45 & 26 & Partially laminated \\
\hline $3 \mathrm{H}-1,54-69$ & 16.54 & 45 & 27 & Laminated \\
\hline $3 \mathrm{H}-1,129-144$ & 17.29 & 45 & 28 & Laminated \\
\hline $3 \mathrm{H}-2,1-16$ & 17.51 & 45 & 29 & Partially laminated \\
\hline $3 \mathrm{H}-4,101-116$ & 21.51 & 45 & 30 & Laminated \\
\hline $3 \mathrm{H}-5,90-105$ & 22.90 & 45 & $31 *$ & Partially laminated \\
\hline $3 \mathrm{H}-6,1-2 \mathrm{I}$ & 23.51 & 60 & 32 & Partially laminated \\
\hline
\end{tabular}

Note: ${ }^{*}$ indicates slabs presented in Table 2.

single years of deposition (Soutar and Crill, 1977), and are regarded as annual varves.

Contact prints with highest resolution were selected for visual inspection and description of the sediment structure. Varves and other distinct layers were counted from bottom to top of a slab on each print and the varve thickness was measured using a hand lens with an integrated scale (graduated to $0.1 \mathrm{~mm}$ ). Where possible, the varve thickness was obtained from the mean of two measurements taken near the left and right margins of an X-radiograph. Out of the 32 slabs (Table 1), we selected seven to be presented here. Table 2 provides detailed description of the stratigraphic character of the 7 slabs, including the quality of definition of varve boundaries. X-radiograph contact prints in Figures 1-3 display the sediment structure. Slabs from Sections 146-893-1 $\mathrm{H}-1$ and $1 \mathrm{H}-2$, were correlated with corresponding intervals of the existing SBB varve chronologies of Lange et al. (in press; "SABA COMPOSITE" in Fig. 1) and Byrne et al. (unpubl. data; "CORE P-2" in Fig. 2), respectively. No previous chronology exists to match slabs 20,23, and 31 presented in Figure 3. In Figures 1 and 2 the visual correlations are based on the comparison and matching of distinct stratigraphic patterns of varve sequences that can be identified from each slab. The cross-correlation of varve records within the 18 th and 17 th centuries (Fig. 1) offers reliable dating to within \pm 10 years, whereas the dating of older varve records in Figure 2 is considered to be less accurate.

We infer that about $30 \mathrm{~cm}$ of the unconsolidated sediment closest to the sediment/water interface (with water contents ranging between 80 to $95 \mathrm{wt} \%$; Schimmelmann et al., 1990) were lost in the coring process. The topmost recovered sediment from Hole 893A was a sludge that did not permit subsampling. However, at about $50-\mathrm{cm}$ depth in Section 146-893-1H-1, below an obviously laminated, albeit physically disturbed sediment interval, we observed an articulated pelecypod, Macoma leptonoidea, which we believe to be associated with a Macoma shell layer in the SBB. This layer is typically located at 55 to $60 \mathrm{~cm}$ below the seafloor and dates back to 1840 A.D.
(Schimmelmann et al., 1992). In Section 146-893-1H-1 the occurrence of the Macoma shell bed is also spaced properly with regard to the distinct non-varved olive layer dated at 1738 A.D. (Lange et al., in press) that is visible at the bottom of our topmost slab 1 (Fig. 1A) and which comprises the first stratigraphic unit in the sequence of slab 1 (Table 2).

\section{SUMMARY}

In addition to a detailed description of the varve stratigraphy, we present X-radiographic and other evidence for reliable cross-correlations of selected varved intervals of Sections $1 \mathrm{H}-1$ through $1 \mathrm{H}-3$ with previously established and dated SBB varve records. The find of a diagnostic Macoma shell bed near the top of Section 1H-1 suggests that Hole $893 \mathrm{~A}$ lost about $30 \mathrm{~cm}$ of unconsolidated sediment below the 1992 sediment/water interface during the coring process. The general state of compaction and dewatering of the sediment of Section $1 \mathrm{H}-1$ also implies that it derives from about $65 \mathrm{~cm}$ below the 1992 seafloor. Hole $893 \mathrm{~A}$ provides a useful varve record with ages as young as the late 18 th century.

\section{ACKNOWLEDGMENTS}

We thank Tim Baumgartner and William Busch for critical reviews of the manuscript and Susan Green of the Scripps Photo Lab for endless hours in the dark room. This study was supported in part by NSF grant OCE93-01438.

\section{REFERENCES}

Baumgartner, T.R., Ferreira-Bartrina, V., Cowen, J., and Soutar, A., 1991. Reconstruction of a twentieth century varve chronology from the central 
Gulf of California. In Dauphin, J.P., and Simoneit, B.R.T. (Eds.), The Gulf and Peninsular Province of the Californias. AAPG Mem., 47:603616.

Baumgartner, T.R., Michaelsen, J., Thompson, L.G., Shen, G.T., Soutar, A., and Casey, R.E., 1989. The recording of interannual climatic change by high-resolution natural systems: tree-rings, coral bands, glacial ice layers, and marine varves. In Peterson, D.H. (Ed.), Aspects of Climate Variability in the Pacific and the Western Americas. U.S. Geol. Surv., Geophys. Monogr., 55:1-14.

Emery, K.O., and Hülsemann, J., 1962. The relationships of sediments, lifeand water in a marine basin. Deep-Sea Res. Part A, 8:165-180.

Lange, C.B., Schimmelmann, A., Yasuda, M.K., and Berger, W.H., in press. Paleoclimatic significance of marine varves off southern California. In Wigand, P., and Rose, M. (Eds.), Southern California Climate: Trends and Extremes of the Past 2000 Years: Los Angeles (Los Angeles County Museum of Natural History).

Reimers, C.E., Lange, C.B., Tabak, M., and Bernhard, J.M., 1990. Seasonal spillover and varve formation in the Santa Barbara Basin, California. Limnol. Oceanogr., 35:1577-1585.

Schimmelmann, A., Lange, C.B., and Berger, W.H., 1990. Climatically controlled marker layers in Santa Barbara basin sediments, and fine-scale core-to-core correlation. Limnol. Oceanogr., 35:165-173.
Schimmelmann, A., Lange, C.B., Berger, W.H., Simon, A., Burke, S.K., and Dunbar, R.B., 1992. Extreme climatic conditions recorded in Santa Barbara basin laminated sediments: the 1835-1840 Macoma event. Mar. Geol., 106:279-299.

Shore-based Scientific Party, 1994. Site 893. In Kennett, J.P., Baldauf, J.G., et al., 1994. Proc. ODP, Init. Repts., 146 (Pt. 2): College Station, TX (Ocean Drilling Program), 15-81.

Soutar, A., and Crill, P.A., 1977. Sedimentation and climatic patterns in the Santa Barbara Basin during the 19th and 20th centuries. Geol. Soc. Am. Bull., 88:1161-1172.

Soutar, A., Johnson, S.R., Taylor, E., and Baumgartner, T.R., 1982. X-radiography of Hole 480: procedures and results. In Curray, J.R., Moore, D.G., et al., Init. Repts. DSDP, 64 (Pt. 2): Washington (U.S. Govt. Printing Office), 1183-1190.

Thornton, S.E., 1986. Origin of mass flow sedimentary structures in hemipelagic basin deposits: Santa Barbara basin. California borderland. GeoMar. Lett. 6:15-19.

Date of initial receipt: 17 August 1994

Date of acceptance: 14 February 1995

Ms 146SR-272 
CORE 1H-1

SLAB 1

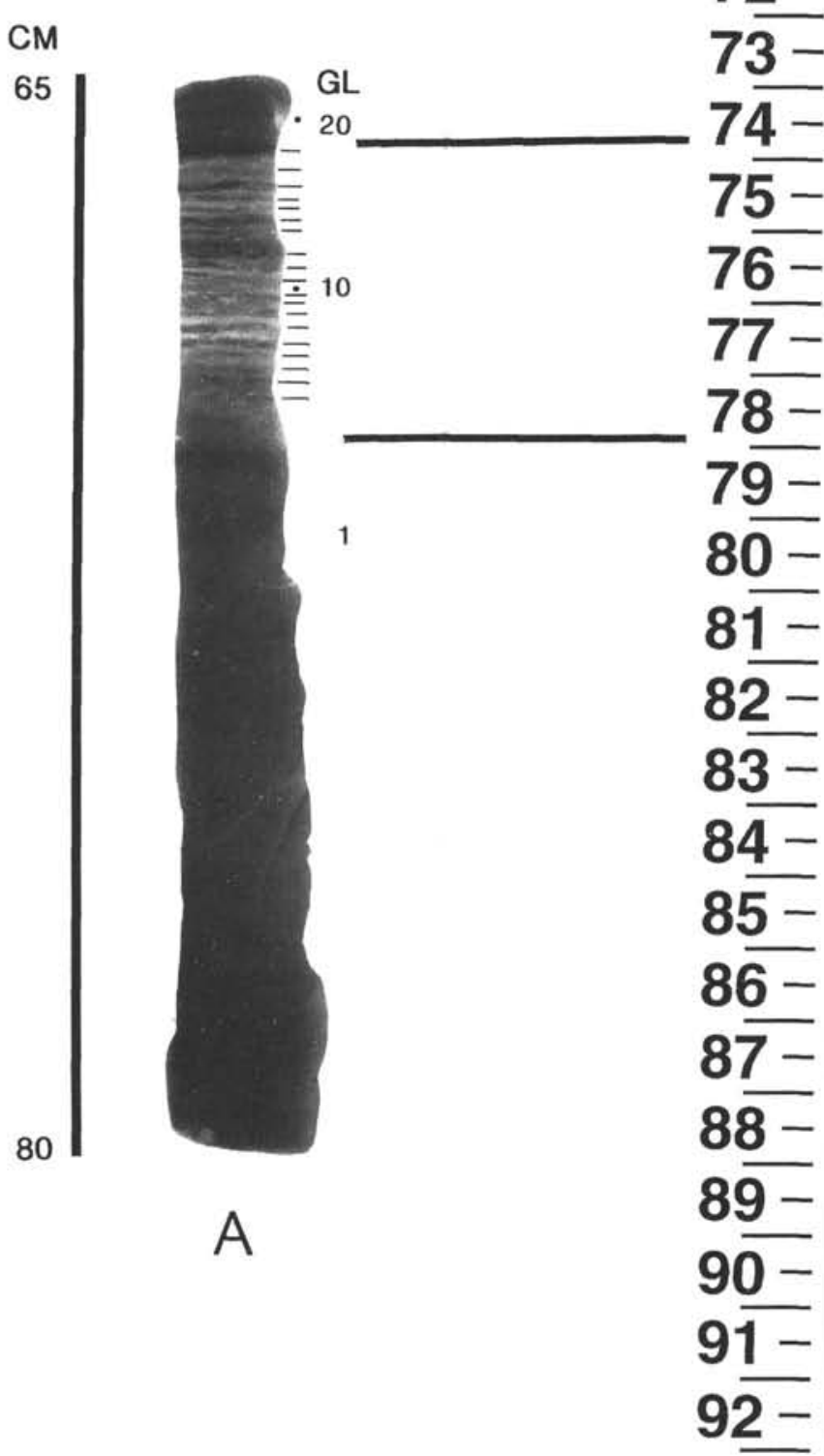

\section{Depth}

in Core

(cm)

\section{SABA \\ COMPOSITE}

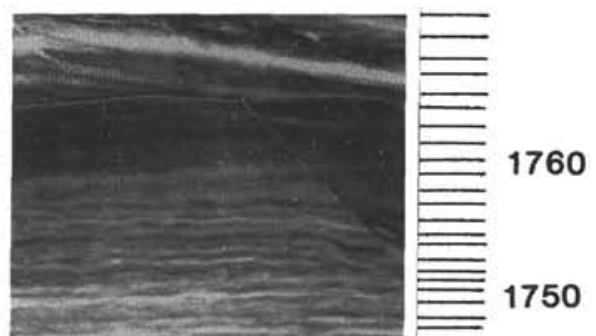

1750

1740
GL

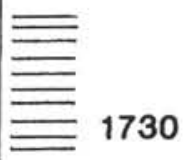

Year A.D.

Figure 1. X-radiograph contact prints of (A) slab 1, Sample 146-893A-1H-1, 65-80 cm, and (B) slab 2, Sample 146-893A-1H-1, 100-115 cm, are shown beside comparable sections of previously dated Santa Barbara Basin varve records. The "SABA COMPOSITE" X-radiograph varve chronology was obtained from nearby Santa Barbara Basin locations (Lange et al., in press). Distinct sediment layers (varves, bioturbated layers, turbidites, and intervals with unresolved or missing lamination) are numbered sequentially from bottom to top of slabs of Section 146-983A-1H-1, with their boundaries indicated by tickmarks at one side of X-radiograph contact prints (see also Table 2). Layers \#10, 20,30, etc., are additionally identified by dots between tickmarks. The vertical length of slabs and the corresponding depth intervals within each section (Table 1) are indicated by black bars for the 893A slabs. A scale indicating cm below seafloor is given for each SABA COMPOSITE slab. Correlation lines indicate the inferred correspondence between the 893A and SABA cores. GL $=$ gray layer of terrigenous redeposited sediment, probably a turbidite (Thornton, 1986), overlain by a non-varved olive layer dated 1738 A.D. 
CORE 1H-1

SLAB 2

CM

100

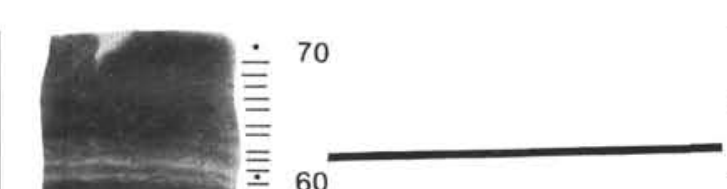

115

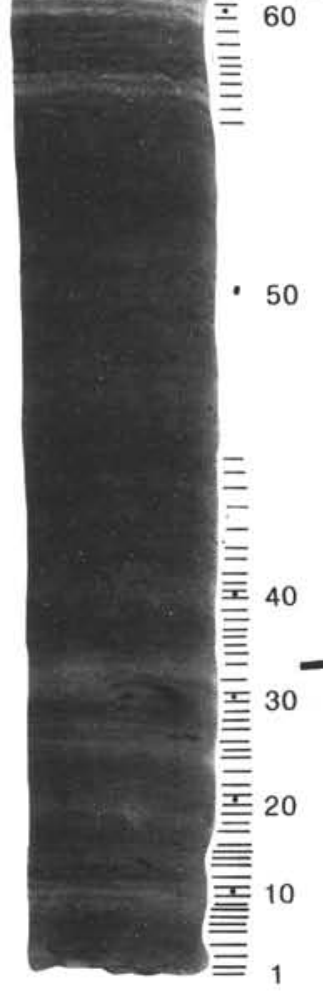

B

SABA

\section{COMPOSITE}

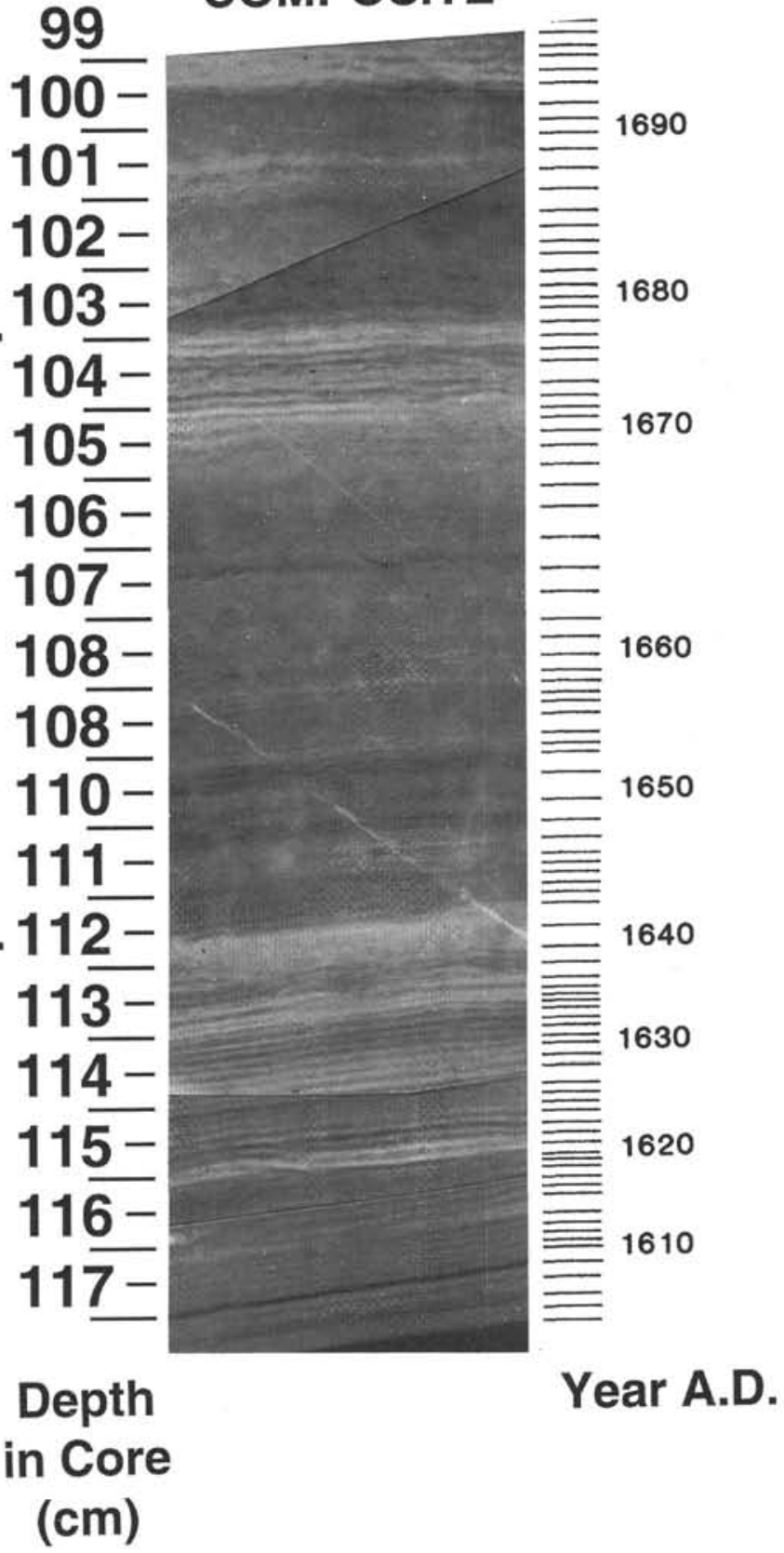

Figure I (continued). 
CORE 1H-2

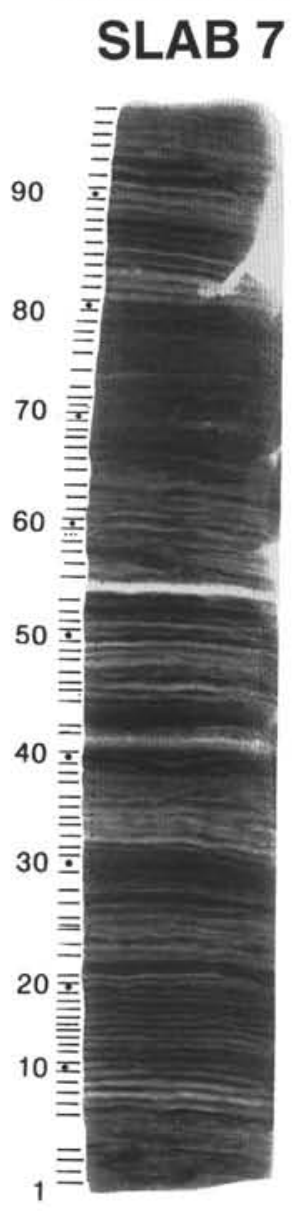

A

CORE P-2

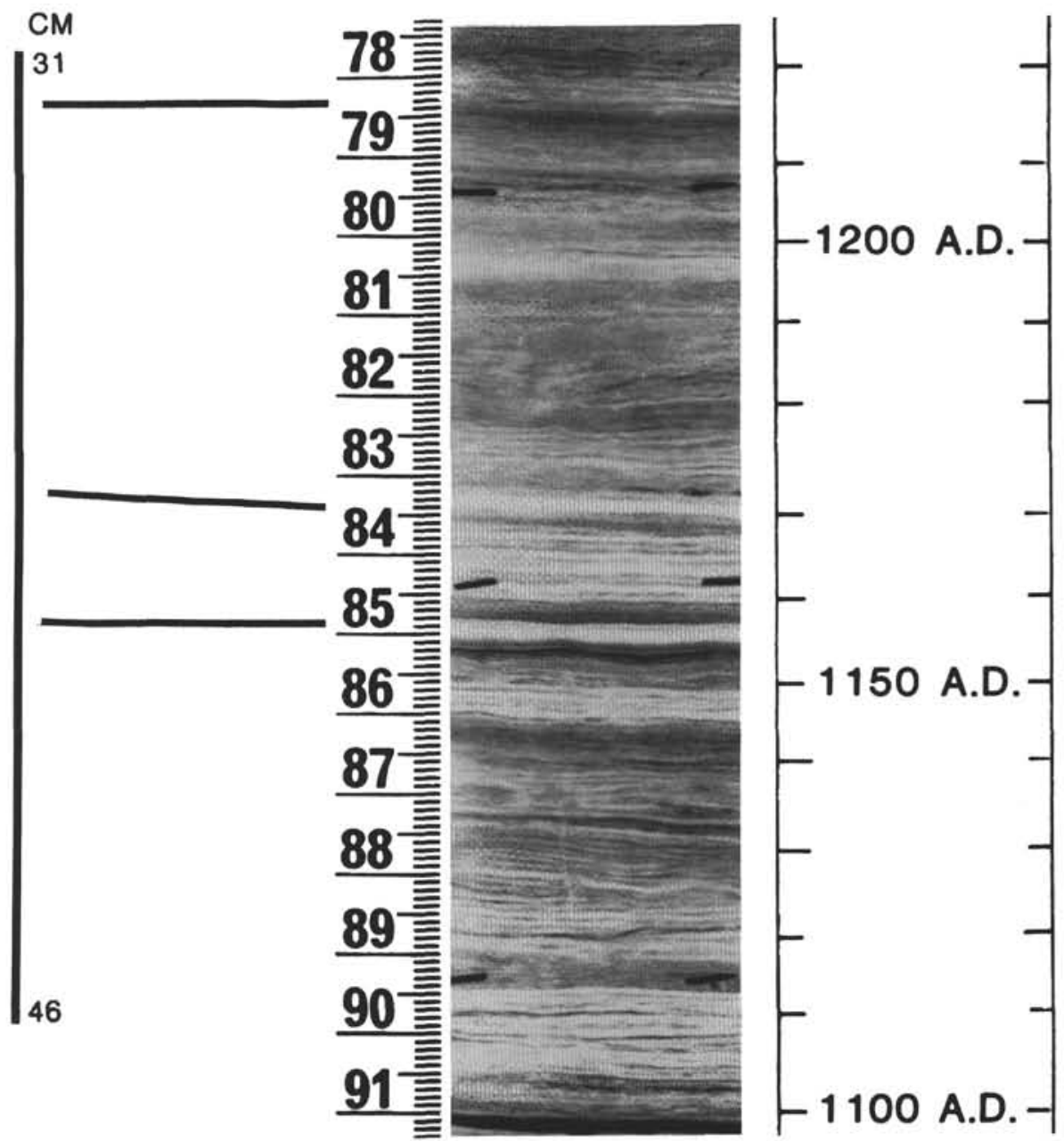

\section{Depth \\ in Core \\ (cm)}

Figure 2. X-radiograph contact prints of (A) slab 7, Sample 146-893A-1H-2, 31-46 cm, and (B) slab 12, Sample 146-893A-1H-2, 109-124 cm, are shown beside comparable sections of previously dated Santa Barbara Basin varve records at a nearby location (Core P- $2 ; 34^{\circ} 14^{\prime} \mathrm{N}, 120^{\circ} 00^{\prime} \mathrm{W}$; dates are approximate). Prints and data from Core P-2 are courtesy of Roger Byrne, UC Berkeley (pers. comm., 1992). Correlation lines indicate the inferred correspondence between slabs of $893 \mathrm{~A}$ and P-2 core. For additional explanations, see Figure 1 caption. 


\section{CORE P-2}

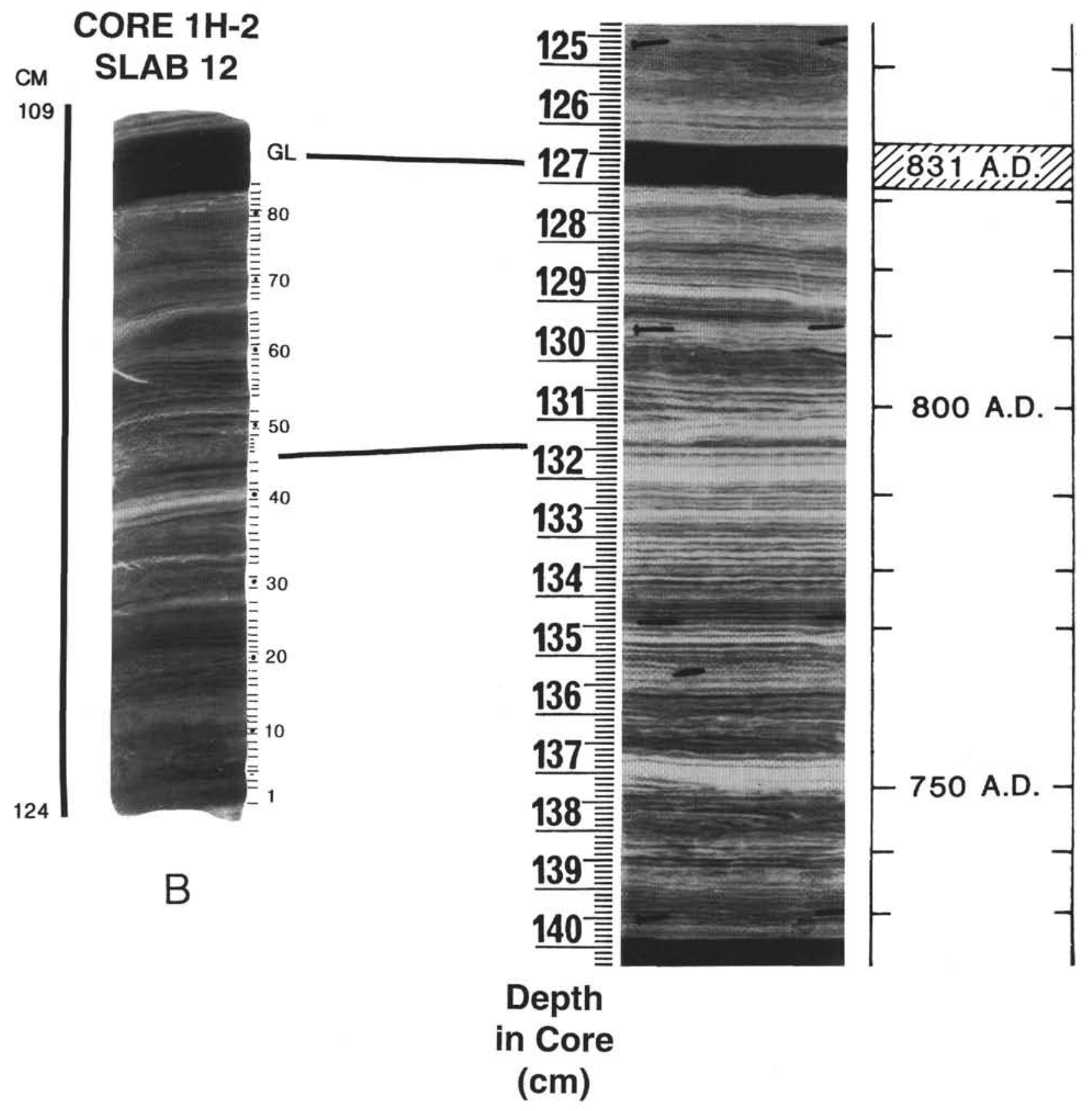

Figure 2 (continued). 

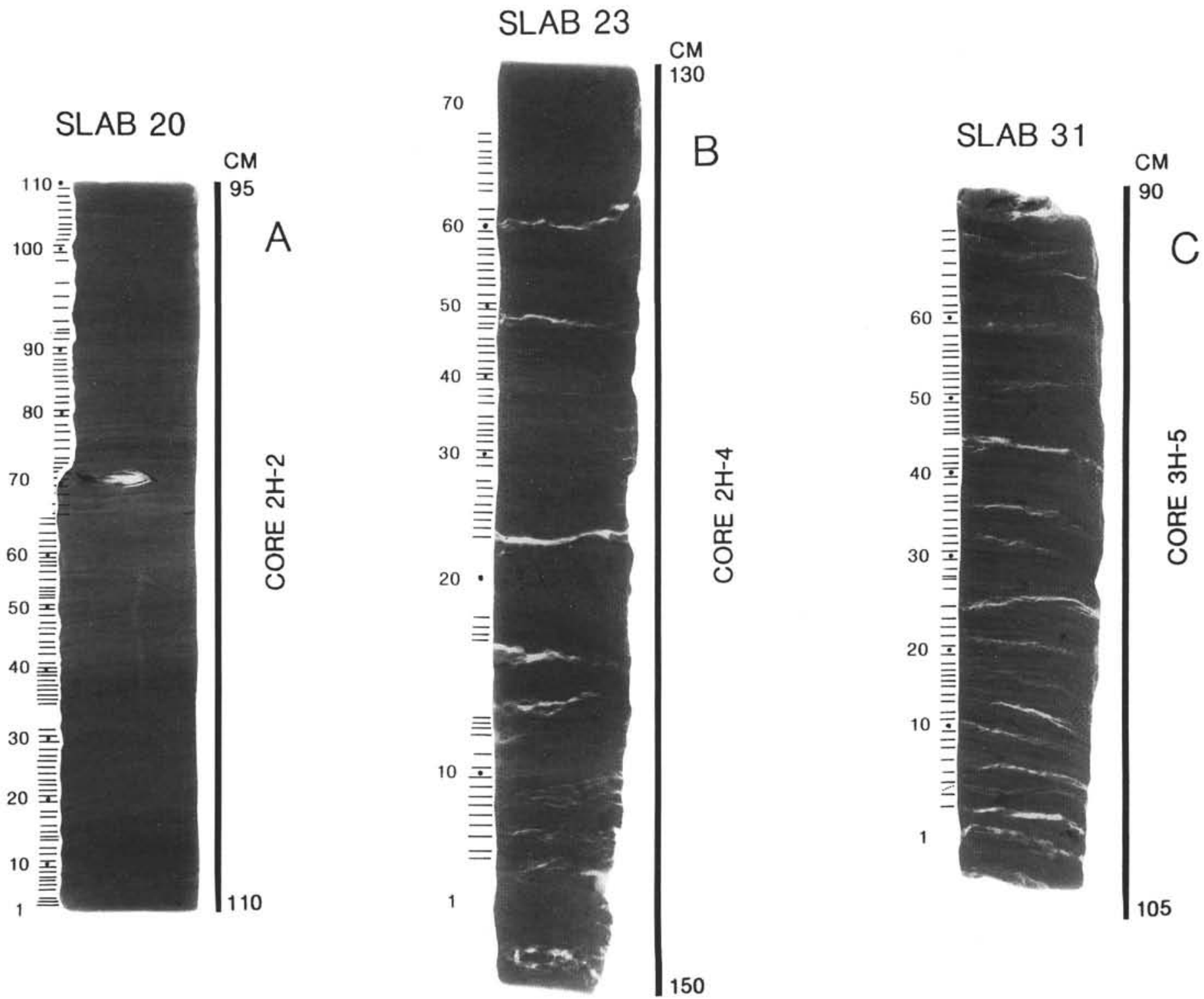

Figure 3. X-radiograph contact prints of (A) slab 20, Sample 146-893A-2H-2, 95-110 cm, (B) slab 23, Sample 146-893A-2H-4, 130-150 cm, and (C) slab 31, Sample 146-893A-3H-5, 90-105 cm. Note the articulated shell in slab 20. Intervals with unresolved lamination are present in slab 23 ("layers" I, 16, 20, and 70) and in slab 31 ("layers" 1 and 69; see Tables 1 and 2 for details). White uneven fissures in slabs 23 and 31 indicate voids generated by methane expansion upon core decompression. For additional explanations, see Figure I caption. 
Table 2. Stratigraphic character of the Hole 893A sediment slabs 1, 2, 7, 12, 20, 23, and 31, including the clarity of the definition of varve boundaries.

\begin{tabular}{|c|c|c|c|c|c|c|c|}
\hline & Core, section, & "Varve" & & & lative th & ness $(\mathrm{mn}$ & \\
\hline Slab \# & interval $(\mathrm{cm})$ & unit & Comments & Left & Right & Center & Mean \\
\hline & 146-893A- & & & ( & & & \\
\hline 1 & IH-I, 65-80 & 1 & Nonlaminated sequence & 104.0 & 104,0 & & 104.0 \\
\hline 1 & $1 \mathrm{H}-1,65-80$ & 2 & Varve boundaries unclear & 2.0 & 2.2 & & 2.1 \\
\hline 1 & $1 \mathrm{H}-1,65-80$ & 3 & Varve boundaries unclear & 2.0 & 2.0 & & 2.0 \\
\hline 1 & $1 \mathrm{H}-1,65-80$ & 4 & Well defined varve & 1.5 & 1.5 & & 1.5 \\
\hline i & $1 \mathrm{H}-1,65-80$ & 5 & Well defined varve & 2.0 & 1.8 & & 1.9 \\
\hline 1 & IH-1, 65-80 & 6 & Well defined varve & 1.9 & 1.9 & & 1.9 \\
\hline 1 & IH-I, 65-80 & 7 & Well defined varve & 2.1 & 2.0 & & 2.1 \\
\hline 1 & IH-1, 65-80 & 8 & Well defined varve & 1.6 & 1.9 & & 1.8 \\
\hline 1 & $1 \mathrm{H}-1,65-80$ & 9 & Well defined varve & 2.0 & 1.5 & & 1,8 \\
\hline 1 & $1 \mathrm{H}-1,65-80$ & 10 & Well defined varve & 2.0 & 2.0 & & 2.0 \\
\hline 1 & $1 \mathrm{H}-1,65-80$ & 11 & Well defined varve & 1.5 & 1.8 & & 1.7 \\
\hline I & $1 \mathrm{H}-\mathrm{I}, 65-80$ & 12 & Well defined varve & 1.2 & 1.5 & & 1.4 \\
\hline i & $\mathrm{IH}-\mathrm{I}, 65-80$ & 13 & Well defined varve & 3.2 & 3.0 & & 3.1 \\
\hline 1 & $1 \mathrm{H}-1,65-80$ & 14 & Well defined varve & 1.2 & 1.8 & & 1.5 \\
\hline i & $\mathrm{IH}-1,65-80$ & 15 & Well defined varve & 2.0 & 1.8 & & 1.9 \\
\hline 1 & IH-I, 65-80 & 16 & Well defined varve & 1.3 & 1.3 & & 1.3 \\
\hline 1 & $1 \mathrm{H}-1,65-80$ & 17 & Well defined varve & 1.2 & 1.7 & & 1.5 \\
\hline 1 & $1 \mathrm{H}-1,65-80$ & 18 & Well defined varve & 2.0 & 2.0 & & 2.0 \\
\hline 1 & IH-1, 65-80 & 19 & Well defined varve & 2.0 & 2.5 & & 2.3 \\
\hline 1 & IH-1, 65-80 & 20 & Nonlaminated, gray layer & 11.1 & 11.0 & & 11.1 \\
\hline 2 & $1 \mathrm{H}-1,100-115$ & 1 & Well defined varve & 1.0 & 1.0 & & 1.0 \\
\hline 2 & $1 \mathrm{H}-1,100-115$ & 2 & Well defined varve & 1.0 & 1.0 & & 1.0 \\
\hline 2 & $1 \mathrm{H}-1,100-115$ & 3 & Well defined varve & & & 1.0 & \\
\hline 2 & $1 \mathrm{H}-\mathrm{I}, 100-115$ & 4 & Well defined varve & 1.1 & 1.1 & & 1.1 \\
\hline 2 & $1 \mathrm{H}-\mathrm{I}, 100-115$ & 5 & Well defined varve & 1.2 & 2.0 & & 1.6 \\
\hline 2 & $1 \mathrm{H}-1,100-115$ & 6 & Well defined varve & 1.0 & 1.0 & & 1.0 \\
\hline 2 & $1 \mathrm{H}-1,100-115$ & 7 & Well defined varve & 1.0 & 1.0 & & 1.0 \\
\hline 2 & $1 \mathrm{H}-1,100-115$ & 8 & Well defined varve & 1.0 & 1.0 & & 1.0 \\
\hline 2 & $1 \mathrm{H}-\mathrm{I}, 100-115$ & 9 & Varve boundaries unclear & & & 2.0 & \\
\hline 2 & IH-I, 100-115 & 10 & Varve boundaries unclear & & & 1.0 & \\
\hline 2 & $1 \mathrm{H}-1,100-115$ & 11 & Varve boundaries unclear & & & 0.9 & \\
\hline 2 & $1 \mathrm{H}-1,100-115$ & 12 & Varve boundaries unclear & & 1.8 & & \\
\hline 2 & $1 \mathrm{H}-1,100-115$ & 13 & Varve boundaries unclear & & 1.0 & & \\
\hline 2 & $1 \mathrm{H}-\mathrm{I}, 100-115$ & 14 & Varve boundaries unclear & & 1.0 & & \\
\hline 2 & $1 \mathrm{H}-1,100-115$ & 15 & Varve boundaries unclear & & 1.0 & & \\
\hline 2 & IH-I, 100-115 & 16 & Varve boundaries unclear & & & 1.8 & \\
\hline 2 & $1 \mathrm{H}-1,100-115$ & 17 & Varve boundaries unclear & & & 1.8 & \\
\hline 2 & $1 \mathrm{H}-\mathrm{I}, 100-115$ & is & Varve boundaries unclear & & & 1.0 & \\
\hline 2 & $1 \mathrm{H}-1,100-115$ & 19 & Varve boundaries unclear & & 1.1 & & \\
\hline 2 & $1 \mathrm{H}-\mathrm{I}, 100-115$ & 20 & Varve boundaries unclear & 1.2 & 1.2 & & 1.2 \\
\hline 2 & $1 \mathrm{H}-1,100-115$ & 21 & Varve boundaries unclear & 1.1 & 1.2 & & 1.2 \\
\hline 2 & $1 \mathrm{H}-1,100-115$ & 22 & Unclear, two varves? & & & 2.0 & \\
\hline 2 & IH-1, 100-115 & 23 & Well defined varve & 0.9 & 0.9 & & 0.9 \\
\hline 2 & $1 \mathrm{H}-1.100-115$ & 24 & Well defined varve & 0.9 & 0.9 & & 0.9 \\
\hline 2 & $1 \mathrm{H}-\mathrm{I}, 100-115$ & 25 & Well defined varve & 1.5 & 1.5 & & 1.5 \\
\hline 2 & $1 \mathrm{H}-1,100-115$ & 26 & Well defined varve & 1.0 & 1.0 & & 1.0 \\
\hline 2 & $1 \mathrm{H}-1,100-115$ & 27 & Well defined varve & 0.9 & 0.9 & & 0.9 \\
\hline 2 & $1 \mathrm{H}-\mathrm{I}, 100-115$ & 28 & Well defined varve & 1.1 & 1.1 & & 1.1 \\
\hline 2 & $1 \mathrm{H}-1,100-115$ & 29 & Varve boundaries unclear & & 1.0 & & \\
\hline 2 & $1 \mathrm{H}-1,100-115$ & 30 & Varve boundaries unclear & & 1.8 & & \\
\hline 2 & $1 \mathrm{H}-1,100-115$ & 31 & Varve boundaries unclear & & 1.8 & & \\
\hline 2 & $1 \mathrm{H}-1,100-115$ & 32 & Varve boundaries unclear & 1.0 & 1.0 & & 1.0 \\
\hline 2 & $\mathrm{IH}-\mathrm{I}, 100-115$ & 33 & Varve boundaries unclear & 1.0 & 1.0 & & 1.0 \\
\hline 2 & $1 \mathrm{H}-1,100-115$ & 34 & Varve boundaries unclear & & & 1.5 & \\
\hline 2 & $1 \mathrm{H}-1,100-115$ & 35 & Partial varve, not continuous & & 1.1 & & \\
\hline 2 & $1 \mathrm{H}-1,100-115$ & 36 & Partial varve, not continuous & & & 1.0 & \\
\hline 2 & $1 \mathrm{H}-1,100-115$ & 37 & Varve boundaries unclear & & & 1.2 & \\
\hline 2 & $1 \mathrm{H}-1,100-115$ & 38 & Varve boundaries unclear & & & 0.9 & \\
\hline 2 & $1 \mathrm{H}-1,100-115$ & 39 & Varve boundaries unclear & & & 1.2 & \\
\hline 2 & $1 \mathrm{H}-1,100-115$ & 40 & Varve boundaries unclear & & & 0.9 & \\
\hline 2 & $1 \mathrm{H}-1,100-115$ & 41 & Varve boundaries unclear & & & 0.9 & \\
\hline 2 & $1 \mathrm{H}-1,100-115$ & 42 & Varve boundaries unclear & & & 0.8 & \\
\hline 2 & $1 \mathrm{H}-1,100-115$ & 43 & Varve boundaries unclear & & & 1.2 & \\
\hline 2 & $1 \mathrm{H}-1,100-115$ & 44 & Varve boundaries unclear & 1.1 & & & \\
\hline 2 & $1 \mathrm{H}-1,100-115$ & 45 & Seems bioturbated & & & 2.5 & \\
\hline 2 & $1 \mathrm{H}-1,100-115$ & 46 & Seems bioturbated & & & 3.5 & \\
\hline 2 & $1 \mathrm{H}-1,100-115$ & 47 & Seems bioturbated & & & 1.8 & \\
\hline 2 & $1 \mathrm{H}-1,100-115$ & 48 & Seems bioturbated & & & 2.0 & \\
\hline 2 & $1 \mathrm{H}-1,100-115$ & 49 & Varve boundaries unclear & & & 2.0 & \\
\hline 2 & $1 \mathrm{H}-1,100-115$ & 50 & Nonlaminated sequence & 45.0 & 44.5 & & 44.8 \\
\hline 2 & $\mathrm{IH}-1,100-115$ & 51 & Varve boundaries unclear & 1.5 & 1.5 & & 1.5 \\
\hline 2 & $1 \mathrm{H}-1,100-115$ & 52 & Varve boundaries unclear & 1.1 & 1.3 & & 1.2 \\
\hline 2 & $1 \mathrm{H}-1,100-115$ & 53 & Well defined varve & 1.1 & 1.3 & & 1.2 \\
\hline 2 & $1 \mathrm{H}-1,100-115$ & 54 & Well defined varve & 1.2 & 1.2 & & 1.2 \\
\hline 2 & $1 \mathrm{H}-1,100-115$ & 55 & Well defined varve & 1.3 & 1.2 & & 1.3 \\
\hline 2 & $\mathrm{IH}-1,100-115$ & 56 & Well defined varve & 1.5 & 1.0 & & 1.3 \\
\hline 2 & $1 \mathrm{H}-1,100-115$ & 57 & Well defined varve & 1.1 & 1.1 & & 1.1 \\
\hline 2 & $1 \mathrm{H}-1,100-115$ & 58 & Well defined varve & 1.2 & 1.2 & & 1.2 \\
\hline 2 & $1 \mathrm{H}-1,100-115$ & 59 & Well defined varve & 1.5 & 1.5 & & 1.5 \\
\hline 2 & $1 \mathrm{H}-1,100-115$ & 60 & Well defined varve & 1.5 & 1.5 & & 1.5 \\
\hline 2 & $1 \mathrm{H}-1,100-115$ & 61 & Well defined varve & 1.1 & 1.2 & & 1.2 \\
\hline 2 & IH-I, 100-115 & 62 & Well defined varve & 0.5 & 0.8 & & 0.7 \\
\hline 2 & $1 \mathrm{H}-1,100-115$ & 63 & Well defined varve & 2.8 & 1.9 & & 2.4 \\
\hline 2 & $1 \mathrm{H}-1,100-115$ & 64 & Varve boundaries unclear & & & 2.0 & \\
\hline 2 & $1 \mathrm{H}-1,100-115$ & 65 & Varve boundaries unclear & & & 2.0 & \\
\hline 2 & $\mathrm{IH}-1,100-115$ & 66 & Varve boundaries unclear & & & 2.0 & \\
\hline 2 & $1 \mathrm{H}-\mathrm{I}, 100-115$ & 67 & Varve boundaries unclear & & & 1.3 & \\
\hline
\end{tabular}


Table 2 (continued).

\begin{tabular}{|c|c|c|c|c|c|c|c|}
\hline & Core, section, & "Varve" & & & ative th & ness (mr & \\
\hline Slab \# & interval (cm) & unit & Comments & Left & Right & Center & Mean \\
\hline 2 & $1 \mathrm{H}-1,100-115$ & 68 & Varve boundaries unclear & & & 1.0 & \\
\hline 2 & $1 \mathrm{H}-1,100-115$ & 69 & Varve boundaries unclear & & & 5.0 & \\
\hline 7 & $1 \mathrm{H}-2,31-46$ & 1 & Partial varve, not continuous & 1.3 & & & \\
\hline 7 & $1 \mathrm{H}-2,31-46$ & 2 & Varve boundaries unclear & 1.6 & 1.6 & & 1.6 \\
\hline 7 & IH- $2,31-46$ & 3 & Varve boundaries unclear & 1.4 & 1.3 & & 1.4 \\
\hline 7 & $1 \mathrm{H}-2,31-46$ & 4 & Varve boundaries unclear & 2.7 & 1.3 & & 2.0 \\
\hline 7 & $1 \mathrm{H}-2,31-46$ & 5 & Varve boundaries unclear & 3.0 & 4.5 & & 3.8 \\
\hline 7 & $1 \mathrm{H}-2,31-46$ & 6 & Well defined varve & 1.6 & 1.6 & & 1.6 \\
\hline 7 & $\mid \mathrm{H}-2,31-46$ & 7 & Well defined varve & 1.3 & 1.4 & & 1.4 \\
\hline 7 & $1 \mathrm{H}-2,3 \mathrm{I}-46$ & 8 & Well defined varve & 1.7 & 1.4 & & 1.6 \\
\hline 7 & $1 \mathrm{H}-2,31-46$ & 9 & Well defined varve & 1.4 & 1.4 & & 1.4 \\
\hline 7 & $\mid \mathrm{H}-2,3 \mathrm{I}-46$ & 10 & Well defined varve & 1.4 & 1.5 & & 1.5 \\
\hline 7 & $\mathrm{IH}-2,3 \mathrm{I}-46$ & 11 & Well defined varve & 1.5 & 1.1 & & 1.3 \\
\hline 7 & $\mathrm{IH}-2,3 \mathrm{I}-46$ & 12 & Well defined varve & 1.4 & 1.0 & & 1.2 \\
\hline 7 & IH- $2,31-46$ & 13 & Well defined varve & 1.0 & 1.0 & & 1.0 \\
\hline 7 & $\mathrm{IH}-2,3 \mathrm{I}-46$ & 14 & Well defined varve & 1.0 & 1.5 & & 1.3 \\
\hline 7 & $\mathrm{IH}-2,3 \mathrm{I}-46$ & 15 & Well defined varve & 1.0 & 1.0 & & 1.0 \\
\hline 7 & IH- $2,31-46$ & 16 & Well defined varve & 1.0 & 1.0 & & 1.0 \\
\hline 7 & $1 \mathrm{H}-2,3 \mathrm{I}-46$ & 17 & Well defined varve & 1.0 & 1.0 & & 1.0 \\
\hline 7 & $1 \mathrm{H}-2,31-46$ & 18 & Well defined varve & 1.1 & 1.3 & & 1.2 \\
\hline 7 & $\mathrm{IH}-2,3 \mathrm{I}-46$ & 19 & Well defined varve & 1.0 & 0.9 & & 1.0 \\
\hline 7 & $1 \mathrm{H}-2,31-46$ & 20 & Well defined varve & 1.1 & 2.1 & & 1.6 \\
\hline 7 & $1 \mathrm{H}-2,31-46$ & 21 & Partial varve, not continuous & 1.1 & & & \\
\hline 7 & $1 \mathrm{H}-2,31-46$ & 22 & Well defined varve & 2.4 & 2.5 & & 2.5 \\
\hline 7 & $\mid \mathrm{H}-2,31-46$ & 23 & Well defined varve & 1.5 & 1.4 & & 1.5 \\
\hline 7 & $\mid \mathrm{H}-2,3 \mathrm{I}-46$ & 24 & Well defined varve & 1.5 & 1.3 & & 1.4 \\
\hline 7 & $\mathrm{IH}-2,31-46$ & 25 & Well defined varve & 1.3 & 1.0 & & 1.2 \\
\hline 7 & $\mathrm{IH}-2,31-46$ & 26 & Well defined varve & 1.1 & 1.3 & & 1.2 \\
\hline 7 & IH-2, 31-46 & 27 & Well defined varve & 1.2 & 1.2 & & 1.2 \\
\hline 7 & $\mathrm{IH}-2,31-46$ & 28 & Well defined varve & 2.9 & 2.5 & & 2.7 \\
\hline 7 & $\mathrm{IH}-2,3 \mathrm{i}-46$ & 29 & Well defined varve & 1.9 & 1.5 & & 1.7 \\
\hline 7 & IH-2, 31-46 & 30 & Well defined varve & 2.0 & 3.2 & & 2.6 \\
\hline 7 & $\mid \mathrm{H}-2,3 \mathrm{I}-46$ & 31 & Well defined varve & 1.1 & 1.3 & & 1.2 \\
\hline 7 & $1 \mathrm{H}-2,31-46$ & 32 & Well defined varve & 1.7 & 1.7 & & 1.7 \\
\hline 7 & $1 \mathrm{H}-2,31-46$ & 33 & Well defined varve & 1.3 & 1.0 & & 1.2 \\
\hline 7 & IH-2, $31-46$ & 34 & Well defined varve & 0.9 & I.I & & 1.0 \\
\hline 7 & $\mid \mathrm{H}-2,3 \mathrm{I}-46$ & 35 & Well defined varve & 1.3 & 1.6 & & 1.5 \\
\hline 7 & $\mathrm{IH}-2,3 \mathrm{I}-46$ & 36 & Well defined varve & 1.8 & 1.6 & & 1.7 \\
\hline 7 & $\mathrm{IH}-2,31-46$ & 37 & Well defined varve & 1.2 & 1.6 & & 1.4 \\
\hline 7 & $\mathrm{IH}-2,3 \mathrm{I}-46$ & 38 & Well defined varve & 2.0 & 1.7 & & 1.9 \\
\hline 7 & $1 \mathrm{H}-2,31-46$ & 39 & Well defined varve & 1.0 & 1.2 & & 1.1 \\
\hline 7 & $\mid \mathrm{H}-2,31-46$ & 40 & Well defined varve & 1.1 & 1.0 & & 1.1 \\
\hline 7 & $\mathrm{IH}-2,31-46$ & 41 & Unclear, two varves? & 2.4 & 2.4 & & 2.4 \\
\hline 7 & $\mathrm{IH}-2,31-46$ & 42 & Well defined varve & 1.3 & 2.0 & & 1.7 \\
\hline 7 & $1 \mathrm{H}-2,31-46$ & 43 & Thin gray layer & 3.5 & 2.5 & & 3.0 \\
\hline 7 & $\mathrm{IH}-2,3 \mathrm{I}-46$ & 44 & Well defined varve & 1.3 & 1.2 & & 1.3 \\
\hline 7 & $1 \mathrm{H}-2,3 \mathrm{i}-46$ & 45 & Well defined varve & 1.2 & 1.1 & & 1.2 \\
\hline 7 & $1 \mathrm{H}-2,31-46$ & 46 & Well defined varve & 1.5 & 1.7 & & 1.6 \\
\hline 7 & $1 \mathrm{H}-2,3 \mathrm{i}-46$ & 47 & Well defined varve & 1.3 & 1.4 & & 1.4 \\
\hline 7 & $1 \mathrm{H}-2,31-46$ & 48 & Well defined varve & 1.1 & 1.0 & & 1.1 \\
\hline 7 & $1 \mathrm{H}-2,31-46$ & 49 & Well defined varve & 1.1 & 1.4 & & 1.3 \\
\hline 7 & $\mid \mathrm{H}-2,3 \mathrm{I}-46$ & 50 & Well defined varve & 1.6 & 1.6 & & 1.6 \\
\hline 7 & IH-2, $31-46$ & 51 & Well defined varve & 1.5 & 1.4 & & 1.5 \\
\hline 7 & $1 \mathrm{H}-2,31-46$ & 52 & Well defined varve & 1.0 & 1.0 & & 1.0 \\
\hline 7 & $1 \mathrm{H}-2,31-46$ & 53 & Well defined varve & 1.7 & 1.6 & & 1.7 \\
\hline 7 & $1 \mathrm{H}-2,31-46$ & 54 & Well defined varve & 2.8 & 2.6 & & 2.7 \\
\hline 7 & $1 \mathrm{H}-2,31-46$ & 55 & Well defined varve & 1.6 & 1.0 & & 1.3 \\
\hline 7 & $1 \mathrm{H}-2,31-46$ & 56 & Partial varve, not continuous & 0.9 & & & \\
\hline 7 & $\mathrm{IH}-2,3 \mathrm{I}-46$ & 57 & Varve boundaries unclear & 1.6 & 1.3 & & 1.5 \\
\hline 7 & $|\mathrm{H}-2,3|-46$ & 58 & Partial varve, not continuous & & 1.4 & & \\
\hline 7 & $1 \mathrm{H}-2,3 \mathrm{I}-46$ & 59 & Varve boundaries unclear & 1.6 & 1.7 & & 1.7 \\
\hline 7 & $1 \mathrm{H}-2,31-46$ & 60 & Well defined varve & 1.5 & 1.3 & & 1.4 \\
\hline 7 & $\mathrm{IH}-2,3 \mathrm{I}-46$ & 61 & Well defined varve & 1.2 & 1.7 & & 1.5 \\
\hline 7 & IH-2, 3I-46 & 62 & Well defined varve & 1.6 & 1.3 & & 1.5 \\
\hline 7 & $1 \mathrm{H}-2,31-46$ & 63 & Well defined varve & 1.7 & 1.7 & & 1.7 \\
\hline 7 & $1 \mathrm{H}-2,31-46$ & 64 & Well defined varve & 2.0 & 2.0 & & 2.0 \\
\hline 7 & IH $-2,31-46$ & 65 & Well defined varve & 1.8 & 1.0 & & 1.4 \\
\hline 7 & $1 \mathrm{H}-2,31-46$ & 66 & Well defined varve & 1.1 & 0.9 & & 1.0 \\
\hline 7 & $1 \mathrm{H}-2,3 \mathrm{I}-46$ & 67 & Well defined varve & 1.0 & 1.5 & & 1.3 \\
\hline 7 & IH $-2,31-46$ & 68 & Well defined varve & 1.4 & 2.6 & & 2.0 \\
\hline 7 & $\mid \mathrm{H}-2,3 \mathrm{I}-46$ & 69 & Varve boundaries unclear & & 1.2 & & \\
\hline 7 & $1 \mathrm{H}-2,31-46$ & 70 & Varve boundaries unclear & & 1.3 & & \\
\hline 7 & $\mathrm{IH}-2,3 \mathrm{I}-46$ & 71 & Varve boundaries unclear & & 1.2 & & \\
\hline 7 & IH-2, 31-46 & 72 & Varve boundaries unclear & & 1.6 & & \\
\hline 7 & $\mid \mathrm{H}-2,3 \mathrm{I}-46$ & 73 & Varve boundaries unclear & & 2.0 & & \\
\hline 7 & $1 \mathrm{H}-2,3 \mathrm{I}-46$ & 74 & Varve boundaries unclear & 1.7 & 2.2 & & 2.0 \\
\hline 7 & $1 \mathrm{H}-2,3 \mathrm{I}-46$ & 75 & Varve boundaries unclear & 2.4 & & & \\
\hline 7 & IH $-2,31-46$ & 76 & Varve boundaries unclear & 1.8 & & & \\
\hline 7 & $1 \mathrm{H}-2,3 \mathrm{I}-46$ & 77 & Well defined varve & 1.0 & 1.1 & & 1.1 \\
\hline 7 & IH-2, $31-46$ & 78 & Well defined varve & 1.3 & 1.1 & & 1.2 \\
\hline 7 & $1 \mathrm{H}-2,31-46$ & 79 & Well defined varve & 1.2 & 1.5 & & 1.4 \\
\hline 7 & $\mathrm{IH}-2,3 \mathrm{I}-46$ & 80 & Well defined varve & 1.2 & 1.1 & & 1.2 \\
\hline 7 & $\mathrm{IH}-2,3 \mathrm{I}-46$ & 81 & Well defined varve & 1.6 & 1.4 & & 1.5 \\
\hline 7 & $\mathrm{IH}-2,3 \mathrm{I}-46$ & 82 & Well defined varve & 1.2 & 1.0 & & 1.1 \\
\hline 7 & $\mid \mathrm{H}-2,3 \mathrm{I}-46$ & 83 & Well defined varve & 1.7 & 1.5 & & 1.6 \\
\hline 7 & IH-2, 31- 46 & 84 & Partial varve, not continuous & 1.3 & & & 0.7 \\
\hline 7 & IH-2, $31-46$ & 85 & Well defined varve & 1.8 & 1.7 & & 1.8 \\
\hline 7 & $\mathrm{IH}-2,31-46$ & 86 & Well defined varve & 1.5 & 1.7 & & 1.6 \\
\hline
\end{tabular}


Table 2 (continued).

\begin{tabular}{|c|c|c|c|c|c|c|c|}
\hline & Core, section, & "Varve" & & & ative thi & ness (mm & \\
\hline Slab \# & interval (cm) & unit & Comments & Left & Right & Center & Mean \\
\hline 7 & $1 \mathrm{H}-2,31-46$ & 87 & Well defined varve & 1.0 & 1.7 & & 1.4 \\
\hline 7 & $1 \mathrm{H}-2,31-46$ & 88 & Well defined varve & 1.8 & 1.8 & & 1.8 \\
\hline 7 & $1 \mathrm{H}-2,31-46$ & 89 & Well defined varve & 1.7 & 1.4 & & 1.6 \\
\hline 7 & $1 \mathrm{H}-2,3 \mathrm{I}-46$ & 90 & Well defined varve & 1.1 & 1.5 & & 1.3 \\
\hline 7 & $1 \mathrm{H}-2,31-46$ & 91 & Well defined varve & 1.6 & 1.8 & & 1.7 \\
\hline 7 & $1 \mathrm{H}-2,3 \mathrm{I}-46$ & 92 & Well defined varve & 2.0 & 2.5 & & 2.3 \\
\hline 7 & $1 \mathrm{H}-2,31-46$ & 93 & Well defined varve & 2.2 & 2.0 & & 2.1 \\
\hline 7 & $1 \mathrm{H}-2,31-46$ & 94 & Well defined varve & 1.5 & 1.5 & & 1.5 \\
\hline 7 & $\mathrm{IH}-2,3 \mathrm{I}-46$ & 95 & Well defined varve & 1.1 & 1.2 & & 1.2 \\
\hline 7 & $1 \mathrm{H}-2,31-46$ & 96 & Well defined varve & 2.0 & 1.4 & & 1.7 \\
\hline 7 & $\mid \mathrm{H}-2,31-46$ & 97 & Well defined varve & 1.5 & 1.3 & & 1.4 \\
\hline 12 & $1 \mathrm{H}-2,109-124$ & 1 & Varve boundaries unclear & 1.6 & 3.4 & & 2.5 \\
\hline 12 & $1 \mathrm{H}-2,109-124$ & 2 & Varve boundaries unclear & 1.0 & 1.3 & & 1.2 \\
\hline 12 & $1 \mathrm{H}-2,109-124$ & 3 & Varve boundaries unclear & 1.0 & 1.4 & & 1.2 \\
\hline 12 & IH-2, $109-124$ & 4 & Varve boundaries unclear & 1.6 & 0.5 & & 1.1 \\
\hline 12 & IH- $2,109-124$ & 5 & Varve boundaries unclear & 1.0 & 0.8 & & 0.9 \\
\hline 12 & IH-2, $109-124$ & 6 & Varve boundaries unclear & & & 2.0 & \\
\hline 12 & IH-2, $109-124$ & 7 & Varve boundaries unclear & & & 1.0 & \\
\hline 12 & IH-2, $109-124$ & 8 & Varve boundaries unclear & & & 1.0 & \\
\hline 12 & $\mathrm{IH}-2,109-124$ & 9 & Varve boundaries unclear & & & 1.4 & \\
\hline 12 & $\mathrm{IH}-2,109-124$ & 10 & Varve boundaries unclear & & & 1.0 & \\
\hline 12 & $1 \mathrm{H}-2,109-124$ & 11 & Varve boundaries unclear & & & 1.0 & \\
\hline 12 & $1 \mathrm{H}-2,109-124$ & 12 & Varve boundaries unclear & & & 1.6 & \\
\hline 12 & $1 \mathrm{H}-2,109-124$ & 13 & Varve boundaries unclear & & & 1.1 & \\
\hline 12 & $1 \mathrm{H}-2,109-124$ & 14 & Varve boundaries unclear & & & 1.3 & \\
\hline 12 & IH-2, $109-124$ & is & Varve boundaries unclear & 1.5 & 1.1 & & 1.3 \\
\hline 12 & $1 \mathrm{H}-2,109-124$ & 16 & Varve boundaries unclear & 1.2 & 2.1 & & 1.7 \\
\hline 12 & $\mathrm{IH}-2,109-124$ & 17 & Well defined varve & 1.3 & 1.3 & & 1.3 \\
\hline 12 & $\mathrm{IH}-2,109-124$ & 18 & Varve boundaries unclear & 1.0 & 1.0 & & 1.0 \\
\hline 12 & $1 \mathrm{H}-2,109-124$ & 19 & Partial varve, not continuous & & 2.1 & & \\
\hline 12 & $\mathrm{IH}-2,109-124$ & 20 & Varve boundaries unclear & 2.2 & 1.5 & & 1.9 \\
\hline 12 & $\mathrm{IH}-2,109-124$ & 21 & Partial varve, not continuous & 1.0 & & & \\
\hline 12 & $1 \mathrm{H}-2,109-124$ & 22 & Varve boundaries unclear & & 0.8 & & \\
\hline 12 & $1 \mathrm{H}-2,109-124$ & 23 & Varve boundaries unclear & & 1.1 & & \\
\hline 12 & $1 \mathrm{H}-2,109-124$ & 24 & Well defined varve & & & 1.1 & \\
\hline 12 & $\mathrm{IH}-2,109-124$ & 25 & Well defined varve & & & 0.9 & \\
\hline 12 & $\mathrm{IH}-2,109-124$ & 26 & Well defined varve & & & 1.2 & \\
\hline 12 & $\mathrm{IH}-2,109-124$ & 27 & Well defined varve & & & 2.2 & \\
\hline 12 & $1 \mathrm{H}-2,109-124$ & 28 & Well defined varve & & & 1.1 & \\
\hline 12 & $1 \mathrm{H}-2,109-124$ & 29 & Well defined varve & 2.8 & 3.2 & & 3.0 \\
\hline 12 & IH-2, 109-124 & 30 & Well defined varve & & 2.1 & & \\
\hline 12 & $1 \mathrm{H}-2,109-124$ & 31 & Well defined varve & & 2.8 & & \\
\hline 12 & $1 \mathrm{H}-2,109-124$ & 32 & Well defined varve & & 1.4 & & \\
\hline 12 & IH-2, 109-124 & 33 & Well defined varve & & 2.2 & & \\
\hline 12 & IH-2, $109-124$ & 34 & Well defined varve & & 1.5 & & \\
\hline 12 & $1 \mathrm{H}-2,109-124$ & 35 & Well defined varve & & 1.5 & & \\
\hline 12 & $\mathrm{IH}-2,109-124$ & 36 & Well defined varve & & 1.4 & & \\
\hline 12 & $1 \mathrm{H}-2,109-124$ & 37 & Well defined varve & & 1.7 & & \\
\hline 12 & $1 \mathrm{H}-2,109-124$ & 38 & Well defined varve & & 1.7 & & \\
\hline 12 & $1 \mathrm{H}-2,109-124$ & 39 & Well defined varve & 2.0 & 1.3 & & 1.7 \\
\hline 12 & $1 \mathrm{H}-2,109-124$ & 40 & Well defined varve & 1.8 & 2.2 & & 2.0 \\
\hline 12 & $\mathrm{IH}-2,109-124$ & 41 & Well defined varve & 1.7 & 1.3 & & 1.5 \\
\hline 12 & $1 \mathrm{H}-2,109-124$ & 42 & Well defined varve & 1.0 & 2.4 & & 1.7 \\
\hline 12 & $\mathrm{IH}-2,109-124$ & 43 & Well defined varve & 2.1 & 1.8 & & 2.0 \\
\hline 12 & $1 \mathrm{H}-2,109-124$ & 44 & Varve boundaries unclear & & 1.6 & & \\
\hline 12 & $1 \mathrm{H}-2,109-124$ & 45 & Varve boundaries unclear & & 0.8 & & \\
\hline 12 & $1 \mathrm{H}-2,109-124$ & 46 & Varve boundaries unclear & & 0.9 & & \\
\hline 12 & $1 \mathrm{H}-2,109-124$ & 47 & Varve boundaries unclear & & 0.6 & & \\
\hline 12 & $1 \mathrm{H}-2,109-124$ & 48 & Varve boundaries unclear & & 1.2 & & \\
\hline 12 & IH-2, 109-124 & 49 & Partial varve, not continuous & & 1.4 & & \\
\hline 12 & $\mathrm{IH}-2,109-124$ & 50 & Well defined varve & 1.5 & 1.3 & & 1.4 \\
\hline 12 & $1 \mathrm{H}-2,109-124$ & 51 & Well defined varve & 1.3 & 1.5 & & 1.4 \\
\hline 12 & $\mathrm{IH}-2,109-124$ & 52 & Varve boundaries unclear & 2.5 & 3.4 & & 3.0 \\
\hline 12 & $1 \mathrm{H}-2,109-124$ & 53 & Well defined varve & 2.2 & 1.4 & & 1.8 \\
\hline 12 & $1 \mathrm{H}-2,109-124$ & 54 & Well defined varve & 1.0 & 1.0 & & 1.0 \\
\hline 12 & $\mathrm{IH}-2,109-124$ & 55 & Well defined varve & 1.4 & 1.1 & & 1.3 \\
\hline 12 & $1 \mathrm{H}-2,109-124$ & 56 & Well defined varve & 1.0 & 1.4 & & 1.2 \\
\hline 12 & IH-2, 109-124 & 57 & Well defined varve & 1.3 & 1.2 & & 1.3 \\
\hline 12 & IH- $2,109-124$ & 58 & Well defined varve & 1.3 & 1.5 & & 1.4 \\
\hline 12 & $1 \mathrm{H}-2,109-124$ & 59 & Well defined varve & 1.2 & 1.0 & & 1.1 \\
\hline 12 & $\mathrm{IH}-2,109-124$ & $60 \mathrm{~A}$ & Well defined varve & 1.3 & 1.2 & & 1.3 \\
\hline 12 & $1 \mathrm{H}-2,109-124$ & $60 \mathrm{~B}$ & Partial varve, not continuous & & 0.7 & & \\
\hline 12 & $\mathrm{IH}-2,109-124$ & 61 & Partial varve, not continuous & & 1.0 & & \\
\hline 12 & $1 \mathrm{H}-2,109-124$ & 62 & Partial varve, not continuous & & 1.2 & & \\
\hline 12 & IH-2, 109-124 & 63 & Varve boundaries unclear & & & 1.7 & \\
\hline 12 & $\mathrm{IH}-2,109-124$ & 64 & Varve boundaries unclear & & & 1.0 & \\
\hline 12 & $1 \mathrm{H}-2,109-124$ & 65 & Varve boundaries unclear & & & 1.0 & \\
\hline 12 & $1 \mathrm{H}-2,109-124$ & 66 & Varve boundaries unclear & & & 2.0 & \\
\hline 12 & IH-2, 109-124 & 67 & Well defined varve & & & 0.9 & \\
\hline 12 & $1 \mathrm{H}-2,109-124$ & 68 & Well defined varve & & & 1.0 & \\
\hline 12 & $1 \mathrm{H}-2,109-124$ & 69 & Well defined varve & & & 1.5 & \\
\hline 12 & $1 \mathrm{H}-2,109-124$ & 70 & Well defined varve & & & 0.9 & \\
\hline 12 & $\mathrm{IH}-2,109-124$ & 71 & Well defined varve & & & 2.2 & \\
\hline 12 & IH-2, 109-124 & 72 & Well defined varve & & & 1.0 & \\
\hline 12 & IH-2, 109-124 & 73 & Well defined varve & & & 1.4 & \\
\hline 12 & IH-2, 109-124 & 74 & Well defined varve & & & 1.1 & \\
\hline 12 & $1 \mathrm{H}-2,109-124$ & 75 & Well defined varve & & & 1.5 & \\
\hline 12 & $1 \mathrm{H}-2,109-124$ & 76 & Well defined varve & & & 1.0 & \\
\hline
\end{tabular}


Table 2 (continued).

\begin{tabular}{|c|c|c|c|c|c|c|}
\hline & section. & "Varve" & & & ative thi & ness (mn \\
\hline Slab \# & interval $(\mathrm{cm})$ & unit & Comments & Left & Right & Center \\
\hline 12 & $1 \mathrm{H}-2,109-124$ & 77 & Well defined varve & & & 1.1 \\
\hline 12 & $1 \mathrm{H}-2,109-124$ & 78 & Well defined varve & & & 1.2 \\
\hline 12 & $1 \mathrm{H}-2,109-124$ & 79 & Partial varve, not continuous & & 0.9 & \\
\hline 12 & $1 \mathrm{H}-2,109-124$ & 80 & Well defined varve & & & 1.3 \\
\hline 12 & $1 \mathrm{H}-2,109-124$ & 81 & Well defined varve & & & 0.9 \\
\hline 12 & $1 \mathrm{H}-2,109-124$ & 82 & Well defined varve & & & 1.1 \\
\hline 12 & IH-2, 109-124 & 83 & Well defined varve & & & 1,1 \\
\hline 12 & $\mathrm{IH}-2,109-124$ & 84 & Well defined varve & & & 1.6 \\
\hline 12 & $1 \mathrm{H}-2,109-124$ & 85 & Nonlaminated, gray layer & 13.0 & 13.0 & \\
\hline 12 & $1 \mathrm{H}-2,109-124$ & 86 & Well defined varve & 0.9 & 1.2 & \\
\hline 12 & $1 \mathrm{H}-2,109-124$ & 87 & Well defined varve & 1.4 & 1.2 & \\
\hline 12 & $1 \mathrm{H}-2,109-124$ & 88 & Well defined varve & 1.1 & 1.9 & \\
\hline 20 & $2 \mathrm{H}-2,95-110$ & 1 & Varve boundaries unclear & & & 1.4 \\
\hline 20 & $2 \mathrm{H}-2,95-110$ & 2 & Varve boundaries unclear & & & 0.9 \\
\hline 20 & $2 \mathrm{H}-2,95-110$ & 3 & Varve boundaries unclear & & & 0.6 \\
\hline 20 & $2 \mathrm{H}-2,95-110$ & 4 & Varve boundaries unclear & & & 1.3 \\
\hline 20 & $2 \mathrm{H}-2,95-110$ & 5 & Well defined varve & & 1.0 & \\
\hline 20 & $2 \mathrm{H}-2,95-110$ & 6 & Well defined varve & & 1.0 & \\
\hline 20 & $2 \mathrm{H}-2,95-110$ & 7 & Well defined varve & & 0.6 & \\
\hline 20 & $2 \mathrm{H}-2,95-110$ & 8 & Well defined varve & & 1.1 & \\
\hline 20 & $2 \mathrm{H}-2,95-110$ & 9 & Well defined varve & & I.1 & \\
\hline 20 & $2 \mathrm{H}-2,95-110$ & 10 & Well defined varve & & 1.2 & \\
\hline 20 & $2 \mathrm{H}-2,95-110$ & II & Well defined varve & & 1.2 & \\
\hline 20 & $2 \mathrm{H}-2,95-110$ & 12 & Well defined varve & & 1.2 & \\
\hline 20 & $2 \mathrm{H}-2,95-110$ & 13 & Well defined varve & & & 1.6 \\
\hline 20 & $2 \mathrm{H}-2,95-110$ & 14 & Partial varve, not continuous & 1.5 & & \\
\hline 20 & $2 \mathrm{H}-2,95-110$ & 15 & Well defined varve & & & 1.0 \\
\hline 20 & $2 \mathrm{H}-2,95-110$ & 16 & Well defined varve & & & 1.0 \\
\hline 20 & $2 \mathrm{H}-2,95-110$ & 17 & Well defined varve & & & 1.4 \\
\hline 20 & $2 \mathrm{H}-2,95-110$ & 18 & Well defined varve & & & 1.4 \\
\hline 20 & $2 \mathrm{H}-2,95-110$ & 19 & Well defined varve & 1.0 & 2.2 & \\
\hline 20 & $2 \mathrm{H}-2,95-110$ & 20 & Well defined varve & 1.2 & 1.0 & \\
\hline 20 & $2 \mathrm{H}-2,95-110$ & 21 & Varve boundaries unclear & & & 1.3 \\
\hline 20 & $2 \mathrm{H}-2,95-110$ & 22 & Varve boundaries unclear & & & 1.3 \\
\hline 20 & $2 \mathrm{H}-2,95-110$ & 23 & Varve boundaries unclear & & & 1.1 \\
\hline 20 & $2 \mathrm{H}-2,95-110$ & 24 & Varve boundaries unclear & & & 1.1 \\
\hline 20 & $2 \mathrm{H}-2,95-110$ & 25 & Varve boundaries unclear & & & 1.1 \\
\hline 20 & $2 \mathrm{H}-2,95-110$ & 26 & Varve boundaries unclear & & & 1.2 \\
\hline 20 & $2 \mathrm{H}-2,95-110$ & 27 & Varve boundaries unclear & & & 1.1 \\
\hline 20 & $2 \mathrm{H}-2,95-110$ & 28 & Varve boundaries unclear & & & 1.2 \\
\hline 20 & $2 \mathrm{H}-2.95-110$ & 29 & Varve boundaries unclear & & & 1.3 \\
\hline 20 & $2 \mathrm{H}-2,95-110$ & 30 & Varve boundaries unclear & & & 0.9 \\
\hline 20 & $2 \mathrm{H}-2,95-110$ & 31 & Varve boundaries unclear & & & 1.2 \\
\hline 20 & $2 \mathrm{H}-2,95-110$ & 32 & Nonlaminated interval & 5.9 & 5.5 & \\
\hline 20 & $2 \mathrm{H}-2,95-110$ & 33 & Partial varve, not continuous & & 0.9 & \\
\hline 20 & $2 \mathrm{H}-2,95-110$ & 34 & Well defined varve & 0.9 & 1.0 & \\
\hline 20 & $2 \mathrm{H}-2,95-110$ & 35 & Well defined varve & 1.2 & 0.9 & \\
\hline 20 & $2 \mathrm{H}-2,95-110$ & 36 & Well defined varve & & & 0.7 \\
\hline 20 & $2 \mathrm{H}-2,95-110$ & 37 & Well defined varve & & & 0.8 \\
\hline 20 & $2 \mathrm{H}-2,95-110$ & 38 & Well defined varve & & & 0.8 \\
\hline 20 & $2 \mathrm{H}-2,95-110$ & 39 & Well defined varve & & & 0.8 \\
\hline 20 & $2 \mathrm{H}-2,95-110$ & 40 & Well defined varve & & & 0.8 \\
\hline 20 & $2 \mathrm{H}-2,95-110$ & 41 & Well defined varve & & & 0.9 \\
\hline 20 & $2 \mathrm{H}-2,95-110$ & 42 & Well defined varve & & & 1.3 \\
\hline 20 & $2 \mathrm{H}-2,95-110$ & 43 & Well defined varve & & & 1.0 \\
\hline 20 & $2 \mathrm{H}-2,95-110$ & 44 & Well defined varve & & & 1.8 \\
\hline 20 & $2 \mathrm{H}-2,95-110$ & 45 & Well defined varve & 1.1 & 1.5 & \\
\hline 20 & $2 \mathrm{H}-2,95-110$ & 46 & Well defined varve & & & 1.4 \\
\hline 20 & $2 \mathrm{H}-2,95-110$ & 47 & Well defined varve & & & 1.4 \\
\hline 20 & $2 \mathrm{H}-2,95-110$ & 48 & Well defined varve & & & 1.7 \\
\hline 20 & $2 \mathrm{H}-2,95-110$ & 49 & Well defined varve & & & 1.2 \\
\hline 20 & $2 \mathrm{H}-2,95-110$ & 50 & Partial varve, not continuous & 1.5 & & \\
\hline 20 & $2 \mathrm{H}-2,95-110$ & 51 & Varve boundaries unclear & 0.7 & 1.1 & \\
\hline 20 & $2 \mathrm{H}-2,95-110$ & 52 & Partial varve, not continuous & & 0.8 & \\
\hline 20 & $2 \mathrm{H}-2,95-110$ & 53 & Partial varve, not continuous & 2.4 & 1.0 & \\
\hline 20 & $2 \mathrm{H}-2,95-110$ & 54 & Varve boundaries unclear & & & \\
\hline 20 & $2 \mathrm{H}-2,95-110$ & 55 & Varve boundaries unclear & & & 1.5 \\
\hline 20 & $2 \mathrm{H}-2,95-110$ & 56 & With pteropod & & & 2.5 \\
\hline 20 & $2 \mathrm{H}-2,95-110$ & 57 & Varve boundaries unclear & & & 1.1 \\
\hline 20 & $2 \mathrm{H}-2,95-110$ & 58 & Varve boundaries unclear & & & 0.8 \\
\hline 20 & $2 \mathrm{H}-2,95-110$ & 59 & Varve boundaries unclear & & & 0.7 \\
\hline 20 & $2 \mathrm{H}-2,95-110$ & 60 & Varve boundaries unclear & & & 1.1 \\
\hline 20 & $2 \mathrm{H}-2,95-110$ & 61 & Varve boundaries unclear & & & 1.2 \\
\hline 20 & $2 \mathrm{H}-2,95-110$ & 62 & Varve boundaries unclear & & & 1.1 \\
\hline 20 & $2 \mathrm{H}-2,95-110$ & 63 & Varve boundaries unclear & & & 2.0 \\
\hline 20 & $2 \mathrm{H}-2,95-110$ & 64 & Varve boundaries unclear & & & 2.4 \\
\hline 20 & $2 \mathrm{H}-2,95-110$ & 65 & Varve boundaries unclear & 1.0 & 2.5 & \\
\hline 20 & $2 \mathrm{H}-2,95-110$ & 66 & Partial varve, not continuous & 0.8 & & \\
\hline 20 & $2 \mathrm{H}-2,95-110$ & 67 & Partial varve, not continuous & & 1.5 & \\
\hline 20 & $2 \mathrm{H}-2,95-110$ & 68 & Well defined varve & 2.6 & 1.9 & \\
\hline 20 & $2 \mathrm{H}-2,95-110$ & 69 & Well defined varve & 1.7 & 1.7 & \\
\hline 20 & $2 \mathrm{H}-2,95-110$ & 70 & Well defined varve & 1.5 & 1.5 & \\
\hline 20 & $2 \mathrm{H}-2,95-110$ & 71 & Well defined varve & & 1.1 & \\
\hline 20 & $2 \mathrm{H}-2,95-110$ & 72 & Well defined varve & & 1.0 & \\
\hline 20 & $2 \mathrm{H}-2,95-110$ & 73 & Well defined varve & & 0.7 & \\
\hline 20 & $2 \mathrm{H}-2,95-110$ & 74 & Well defined varve & & & 0.8 \\
\hline 20 & $2 \mathrm{H}-2,95-110$ & 75 & Well defined varve & 2.1 & 2.0 & \\
\hline 20 & $2 \mathrm{H}-2,95-110$ & 76 & Well defined varve & 2.0 & 2.0 & \\
\hline 20 & $2 \mathrm{H}-2,95-110$ & 77 & Well defined varve & 2.2 & 1.6 & \\
\hline 20 & $2 \mathrm{H}-2,95-110$ & 78 & Well defined varve & 1.3 & 1.1 & \\
\hline
\end{tabular}


Table 2 (continued).

\begin{tabular}{|c|c|c|c|c|c|c|}
\hline & Core, section. & "Varve" & & & ative thi & ness (mn \\
\hline Slab \# & interval $(\mathrm{cm})$ & unit & Comments & Left & Right & Center \\
\hline 20 & $2 \mathrm{H}-2,95-110$ & 79 & Well defined varve & & & 1.9 \\
\hline 20 & $2 \mathrm{H}-2,95-110$ & 80 & Well defined varve & & & 1.1 \\
\hline 20 & $2 \mathrm{H}-2,95-110$ & 81 & Well defined varve & 1.6 & 1.1 & \\
\hline 20 & $2 \mathrm{H}-2,95-110$ & 82 & Well defined varve & & & 1.0 \\
\hline 20 & $2 \mathrm{H}-2,95-110$ & 83 & Varve boundaries unclear & & & 1.5 \\
\hline 20 & $2 \mathrm{H}-2,95-110$ & 84 & Varve boundaries unclear & & & 1.5 \\
\hline 20 & $2 \mathrm{H}-2,95-110$ & 85 & Well defined varve & 1.2 & 1.0 & \\
\hline 20 & $2 \mathrm{H}-2,95-110$ & 86 & Well defined varve & 1.3 & 1.2 & \\
\hline 20 & $2 \mathrm{H}-2,95-110$ & 87 & Well defined varve & 1.0 & 1.2 & \\
\hline 20 & $2 \mathrm{H}-2,95-110$ & 88 & Well defined varve & 0.8 & 0.8 & \\
\hline 20 & $2 \mathrm{H}-2,95-110$ & 89 & Varve boundaries unclear & & & 1.4 \\
\hline 20 & $2 \mathrm{H}-2,95-110$ & 90 & Varve boundaries unclear & & & 1.0 \\
\hline 20 & $2 \mathrm{H}-2,95-110$ & 91 & Varve boundaries unclear & & & 1.7 \\
\hline 20 & $2 \mathrm{H}-2,95-110$ & 92 & Varve boundaries unclear & & 2.4 & \\
\hline 20 & $2 \mathrm{H}-2,95-110$ & 93 & Varve boundaries unclear & & 1.4 & \\
\hline 20 & $2 \mathrm{H}-2.95-110$ & 94 & Varve boundaries unclear & & 1.5 & \\
\hline 20 & $2 \mathrm{H}-2,95-110$ & 95 & Varve boundaries unclear & & & 2.7 \\
\hline 20 & $2 \mathrm{H}-2,95-110$ & 96 & Varve boundaries unclear & & & 2.5 \\
\hline 20 & $2 \mathrm{H}-2,95-110$ & 97 & Varve boundaries unclear & & & 3.0 \\
\hline 20 & $2 \mathrm{H}-2,95-110$ & 98 & Varve boundaries unclear & & & 4.5 \\
\hline 20 & $2 \mathrm{H}-2,95-110$ & 99 & Varve boundaries unclear & & & 1,7 \\
\hline 20 & $2 \mathrm{H}-2,95-110$ & 100 & Well defined varve & & & 1.3 \\
\hline 20 & $2 \mathrm{H}-2,95-110$ & 101 & Well defined varve & & & 1.1 \\
\hline 20 & $2 \mathrm{H}-2,95-110$ & 102 & Well defined varve & & & 1.1 \\
\hline 20 & $2 \mathrm{H}-2,95-110$ & 103 & Well defined varve & & & 1.0 \\
\hline 20 & $2 \mathrm{H}-2.95-110$ & 104 & Well defined varve & & & 1.4 \\
\hline 20 & $2 \mathrm{H}-2,95-110$ & 105 & Well defined varve & & & 1.0 \\
\hline 20 & $2 \mathrm{H}-2,95-110$ & 106 & Well defined varve & & & 1.4 \\
\hline 20 & $2 \mathrm{H}-2,95-110$ & 107 & Well defined varve & & & 1.5 \\
\hline 20 & $2 \mathrm{H}-2,95-110$ & 108 & Well defined varve & & & 1.2 \\
\hline 20 & $2 \mathrm{H}-2,95-110$ & 109 & Well defined varve & & & 1.2 \\
\hline 20 & $2 \mathrm{H}-2,95-110$ & 110 & Well defined varve & & & 1.8 \\
\hline 23 & $2 \mathrm{H}-4,130-150$ & 1 & Nonlaminated sequence & 27.0 & 28.0 & \\
\hline 23 & $2 \mathrm{H}-4,130-150$ & 2 & Varve boundaries unclear & 1.3 & & \\
\hline 23 & $2 \mathrm{H}-4,130-150$ & 3 & Varve boundaries unclear & 2.6 & & \\
\hline 23 & $2 \mathrm{H}-4,130-150$ & 4 & Varve boundaries unclear & 2.6 & & \\
\hline 23 & $2 \mathrm{H}-4,130-150$ & 5 & Varve boundaries unclear & 2.2 & & \\
\hline 23 & $2 \mathrm{H}-4,130-150$ & 6 & Varve boundaries unclear & 1.7 & & \\
\hline 23 & $2 \mathrm{H}-4,130-150$ & 7 & Varve boundaries unclear & 1.8 & & \\
\hline 23 & $2 \mathrm{H}-4,130-150$ & 8 & Varve boundaries unclear & 1.7 & & \\
\hline 23 & $2 \mathrm{H}-4,130-150$ & 9 & Varve boundaries unclear & 2.1 & & \\
\hline 23 & $2 \mathrm{H}-4,130-150$ & 10 & Varve boundaries unclear & 1.5 & & \\
\hline 23 & $2 \mathrm{H}-4,130-150$ & iI & Varve boundaries unclear & 3.2 & & \\
\hline 23 & $2 \mathrm{H}-4,130-150$ & 12 & Varve boundaries unclear & 4.0 & & \\
\hline 23 & $2 \mathrm{H}-4,130-150$ & 13 & Varve boundaries unclear & 1.2 & & \\
\hline 23 & $2 \mathrm{H}-4,130-150$ & 14 & Varve boundaries unclear & 0.9 & & \\
\hline 23 & $2 \mathrm{H}-4,130-150$ & 15 & Varve boundaries unclear & 1.2 & & \\
\hline 23 & $2 \mathrm{H}-4,130-150$ & 16 & Nonlaminated sequence & 16.0 & & \\
\hline 23 & $2 \mathrm{H}-4,130-150$ & 17 & Varve boundaries unclear & 1.1 & & \\
\hline 23 & $2 \mathrm{H}-4,130-150$ & 18 & Varve boundaries unclear & 1.1 & & \\
\hline 23 & $2 \mathrm{H}-4,130-150$ & 19 & Varve boundaries unclear & 2.0 & & \\
\hline 23 & $2 \mathrm{H}-4,130-150$ & 20 & Nonlaminated sequence & 16.5 & 19.0 & \\
\hline 23 & $2 \mathrm{H}-4,130-150$ & 21 & Varve boundaries unclear & 1.5 & & \\
\hline 23 & $2 \mathrm{H}-4,130-150$ & 22 & Varve boundaries unclear & 1.6 & & \\
\hline 23 & $2 \mathrm{H}-4,130-150$ & 23 & Varve boundaries unclear & 1.8 & & \\
\hline 23 & $2 \mathrm{H}-4,130-150$ & 24 & Varve boundaries unclear & 1.2 & & \\
\hline 23 & $2 \mathrm{H}-4,130-150$ & 25 & Varve boundaries unclear & 1.5 & & \\
\hline 23 & $2 \mathrm{H}-4,130-150$ & 26 & Varve boundaries unclear & 1.2 & & \\
\hline 23 & $2 \mathrm{H}-4,130-150$ & 27 & Varve boundaries unclear & 1.6 & & \\
\hline 23 & $2 \mathrm{H}-4,130-150$ & 28 & Varve boundaries unclear & 3.1 & & \\
\hline 23 & $2 \mathrm{H}-4,130-150$ & 29 & Varve boundaries unclear & 1.4 & & \\
\hline 23 & $2 \mathrm{H}-4,130-150$ & 30 & Varve boundaries unclear & 1.6 & & \\
\hline 23 & $2 \mathrm{H}-4,130-150$ & 31 & Varve boundaries unclear & 1.5 & & \\
\hline 23 & $2 \mathrm{H}-4,130-150$ & 32 & Varve boundaries unclear & 1.3 & & \\
\hline 23 & $2 \mathrm{H}-4,130-150$ & 33 & Varve boundaries unclear & 1.4 & & \\
\hline 23 & $2 \mathrm{H}-4,130-150$ & 34 & Varve boundaries unclear & 0.9 & & \\
\hline 23 & $2 \mathrm{H}-4,130-150$ & 35 & Well defined varve & 1.3 & & \\
\hline 23 & $2 \mathrm{H}-4,130-150$ & 36 & Well defined varve & 3.0 & & \\
\hline 23 & $2 \mathrm{H}-4,130-150$ & 37 & Well defined varve & & & 1.6 \\
\hline 23 & $2 \mathrm{H}-4,130-150$ & 38 & Well defined varve & & & 1.6 \\
\hline 23 & $2 \mathrm{H}-4.130-150$ & 39 & Well defined varve & & & 1.7 \\
\hline 23 & $2 \mathrm{H}-4,130-150$ & 40 & Well defined varve & & & 1.6 \\
\hline 23 & $2 \mathrm{H}-4,130-150$ & 41 & Well defined varve & & & 0.9 \\
\hline 23 & $2 \mathrm{H}-4,130-150$ & 42 & Well defined varve & & & 1.4 \\
\hline 23 & $2 \mathrm{H}-4,130-150$ & 43 & Well defined varve & & & 1.3 \\
\hline 23 & $2 \mathrm{H}-4,130-150$ & 44 & Well defined varve & & & 1.2 \\
\hline 23 & $2 \mathrm{H}-4,130-150$ & 45 & Well defined varve & & & 1.2 \\
\hline 23 & $2 \mathrm{H}-4,130-150$ & 46 & Well defined varve & & & 1.7 \\
\hline 23 & $2 \mathrm{H}-4,130-150$ & 47 & Well defined varve & & & 1.4 \\
\hline 23 & $2 \mathrm{H}-4,130-150$ & 48 & Varve boundaries unclear & & & 1.7 \\
\hline 23 & $2 \mathrm{H}-4,130-150$ & 49 & Varve boundaries unclear & & & 2.0 \\
\hline 23 & $2 \mathrm{H}-4,130-150$ & 50 & Well defined varve & 1.3 & & \\
\hline 23 & $2 \mathrm{H}-4,130-150$ & 51 & Well defined varve & 1.6 & & \\
\hline 23 & $2 \mathrm{H}-4,130-150$ & 52 & Well defined varve & 2.4 & & \\
\hline 23 & $2 \mathrm{H}-4,130-150$ & 53 & Well defined varve & 1.0 & & \\
\hline 23 & $2 \mathrm{H}-4,130-150$ & 54 & Well defined varve & 1.5 & & \\
\hline 23 & $2 \mathrm{H}-4,130-150$ & 55 & Well defined varve & 1.6 & & \\
\hline 23 & $2 \mathrm{H}-4,130-150$ & 56 & Well defined varve & 1.7 & & \\
\hline 23 & $2 \mathrm{H}-4,130-150$ & 57 & Well defined varve & 1.1 & & \\
\hline 23 & $2 \mathrm{H}-4,130-150$ & 58 & Well defined varve & 2.2 & & \\
\hline
\end{tabular}


Table 2 (continued).

\begin{tabular}{|c|c|c|c|c|c|c|c|}
\hline \multirow[b]{2}{*}{ Slab \# } & \multirow{2}{*}{$\begin{array}{l}\text { Core, section, } \\
\text { interval }(\mathrm{cm})\end{array}$} & \multirow{2}{*}{$\begin{array}{c}\text { "Varve" } \\
\text { unit }\end{array}$} & \multirow[b]{2}{*}{ Comments } & & ative this & ness (mm & \\
\hline & & & & Left & Right & Center & Mean \\
\hline 23 & $2 \mathrm{H}-4,130-150$ & 59 & Well defined varve & 1.5 & & & \\
\hline 23 & $2 \mathrm{H}-4,130-150$ & 60 & Varve boundaries unclear & 2.0 & & & \\
\hline 23 & $2 \mathrm{H}-4,130-150$ & 61 & Varve boundaries unclear & 2.3 & & & \\
\hline 23 & $2 \mathrm{H}-4,130-150$ & 62 & Could be 2 varves, unclear & 4.0 & & & \\
\hline 23 & $2 \mathrm{H}-4,130-150$ & 63 & Well defined varve & 1.2 & & & \\
\hline 23 & $2 \mathrm{H}-4,130-150$ & 64 & Well defined varve & 2.0 & & & \\
\hline 23 & $2 \mathrm{H}-4,130-150$ & 65 & Well defined varve & 2.0 & & & \\
\hline 23 & $2 \mathrm{H}-4,130-150$ & 66 & Well defined varve & 1.3 & & & \\
\hline 23 & $2 \mathrm{H}-4,130-150$ & 67 & Well defined varve & 1.5 & & & \\
\hline 23 & $2 \mathrm{H}-4,130-150$ & 68 & Well defined varve & 1.5 & & & \\
\hline 23 & $2 \mathrm{H}-4,130-150$ & 69 & Well defined varve & 1.9 & & & \\
\hline 23 & $2 \mathrm{H}-4,130-150$ & 70 & Nonlaminated sequence & 16.0 & 13.5 & & 14.8 \\
\hline 31 & $3 \mathrm{H}-5.90-105$ & 1 & Nonlaminated sequence & 15.0 & 13.0 & & 14.0 \\
\hline 31 & $3 \mathrm{H}-5,90-\mathrm{IOS}$ & 2 & Well defined varve & 2.1 & 1.5 & & 1.8 \\
\hline 31 & $3 \mathrm{H}-5,90-105$ & 3 & Partial varve, not continuous & & 5.0 & & \\
\hline 31 & $3 \mathrm{H}-5,90-105$ & 4 & Partial varve, not continuous & 2.6 & & & \\
\hline 31 & $3 \mathrm{H}-5,90-105$ & 5 & Partial varve, not continuous & 2.0 & & & \\
\hline 31 & $3 \mathrm{H}-5,90-105$ & 6 & Well defined varve & 2.0 & 2.5 & & 2.3 \\
\hline 31 & $3 \mathrm{H}-5,90-105$ & 7 & Well defined varve & 2.4 & 2.4 & & 2.4 \\
\hline 31 & $3 \mathrm{H}-5,90-105$ & 8 & Well defined varve & 2.0 & 1.3 & & 1.7 \\
\hline 31 & $3 \mathrm{H}-5,90-105$ & 9 & Varve boundaries unclear & 3.6 & 1.1 & & 2.4 \\
\hline 31 & $3 \mathrm{H}-5,90-105$ & 10 & Well defined varve & 0.4 & 2.1 & & 1.3 \\
\hline 31 & $3 \mathrm{H}-5,90-105$ & 11 & Varve boundaries unclear & 1.8 & 2.0 & & 1.9 \\
\hline 31 & $3 \mathrm{H}-5,90-\mathrm{I} 05$ & 12 & Varve boundaries unclear & 2.0 & 1.6 & & 1.8 \\
\hline 31 & $3 \mathrm{H}-5,90-105$ & 13 & Well defined varve & 1.2 & 1.5 & & 1.4 \\
\hline 31 & $3 \mathrm{H}-5,90-105$ & 14 & Varve boundaries unclear & 1.9 & 2.1 & & 2.0 \\
\hline 31 & $3 \mathrm{H}-5,90-\mathrm{I} 05$ & 15 & Varve boundaries unclear & 0.8 & 3.1 & & 2.0 \\
\hline 31 & $3 \mathrm{H}-5,90-105$ & 16 & Varve boundaries unclear & 1.2 & 1.0 & & 1.1 \\
\hline 31 & $3 \mathrm{H}-5,90-105$ & 17 & Varve boundaries unclear & 1.0 & 1.2 & & 1.1 \\
\hline 31 & $3 \mathrm{H}-5,90-105$ & 18 & Varve boundaries unclear & 1.2 & 1.4 & & 1.3 \\
\hline 31 & $3 \mathrm{H}-5,90-105$ & 19 & Varve boundaries unclear & 1.6 & 0.5 & & 1.1 \\
\hline $3 i$ & $3 \mathrm{H}-5,90-105$ & 20 & Varve boundaries unclear & 1.5 & 1.5 & & 1.5 \\
\hline 31 & $3 \mathrm{H}-5,90-105$ & 21 & Varve boundaries unclear & 1.5 & 1.3 & & 1.4 \\
\hline 31 & $3 \mathrm{H}-5,90-105$ & 22 & Well defined varve & 1.7 & 1.3 & & 1.5 \\
\hline 31 & $3 \mathrm{H}-5,90-105$ & 23 & Well defined varve & 1.3 & 1.2 & & 1.3 \\
\hline 31 & $3 \mathrm{H}-5,90-105$ & 24 & Well defined varve & 1.9 & 2.0 & & 2.0 \\
\hline 31 & $3 \mathrm{H}-5,90-105$ & 25 & Nonlaminated sequence & 4.4 & 4.7 & & 4.6 \\
\hline 31 & $3 \mathrm{H}-5,90-105$ & 26 & Partial varve, not continuous & 1.6 & & & \\
\hline 31 & $3 \mathrm{H}-5,90-105$ & 27 & Varve boundaries unclear & 1.6 & 2.5 & & 2.1 \\
\hline 31 & $3 \mathrm{H}-5,90-105$ & 28 & Partial varve, not continuous & & 1.9 & & \\
\hline 31 & $3 \mathrm{H}-5,90-105$ & 29 & Varve boundaries unclear & 2.4 & 1.5 & & 2.0 \\
\hline 31 & $3 \mathrm{H}-5,90-105$ & 30 & Varve boundaries unclear & 1.3 & 0.9 & & 1.1 \\
\hline $3 i$ & $3 \mathrm{H}-5,90-105$ & 31 & Varve boundaries unclear & & & 1.5 & \\
\hline 31 & $3 \mathrm{H}-5,90-105$ & 32 & Varve boundaries unclear & & & 1.1 & \\
\hline 31 & $3 \mathrm{H}-5,90-105$ & 33 & Varve boundaries unclear & & & 2.1 & \\
\hline 31 & $3 \mathrm{H}-5,90-105$ & 34 & Varve boundaries unclear & & & 1.4 & \\
\hline 31 & $3 \mathrm{H}-5,90-105$ & 35 & Varve boundaries unclear & & & 1.5 & \\
\hline 31 & $3 \mathrm{H}-5,90-105$ & 36 & Varve boundaries unclear & & & 1.6 & \\
\hline 31 & $3 \mathrm{H}-5,90-105$ & 37 & Varve boundaries unclear & & & 0.8 & \\
\hline 31 & $3 \mathrm{H}-5,90-105$ & 38 & Varve boundaries unclear & & & 1.6 & \\
\hline 31 & $3 \mathrm{H}-5,90-105$ & 39 & Varve boundaries unclear & & & 1.5 & \\
\hline 31 & $3 \mathrm{H}-5,90-105$ & 40 & Varve boundaries unclear & & & 1.5 & \\
\hline 31 & $3 \mathrm{H}-5,90-105$ & 41 & Varve boundaries unclear & & & 1.5 & \\
\hline 31 & $3 \mathrm{H}-5,90-105$ & 42 & Varve boundaries unclear & & & 1.4 & \\
\hline 31 & $3 \mathrm{H}-5,90-105$ & 43 & Varve boundaries unclear & & & 1.5 & \\
\hline 31 & $3 \mathrm{H}-5,90-105$ & 44 & Varve boundaries unclear & 2.9 & 1.7 & & 2.3 \\
\hline 31 & $3 \mathrm{H}-5,90-105$ & 45 & Varve boundaries unclear & & & 1.2 & \\
\hline 31 & $3 \mathrm{H}-5,90-105$ & 46 & Varve boundaries unclear & & & 1.1 & \\
\hline 31 & $3 \mathrm{H}-5,90-105$ & 47 & Varve boundaries unclear & & & 1.4 & \\
\hline 31 & $3 \mathrm{H}-5,90-105$ & 48 & Varve boundaries unclear & & & 0.9 & \\
\hline 31 & $3 \mathrm{H}-5,90-105$ & 49 & Varve boundaries unclear & & & 1.2 & \\
\hline 31 & $3 \mathrm{H}-5,90-105$ & 50 & Varve boundaries unclear & & & 1.7 & \\
\hline 31 & $3 \mathrm{H}-5,90-105$ & 51 & Varve boundaries unclear & 1.2 & 2.3 & & 1.8 \\
\hline 31 & $3 \mathrm{H}-5,90-105$ & 52 & Partial varve, not continuous & 1.7 & & & \\
\hline 31 & $3 \mathrm{H}-5,90-105$ & 53 & Partial varve, not continuous & 1.1 & & & \\
\hline 31 & $3 \mathrm{H}-5,90-105$ & 54 & Varve boundaries unclear & 1.2 & 0.8 & & 1.0 \\
\hline 31 & $3 \mathrm{H}-5,90-105$ & 55 & Varve boundaries unclear & & & 1.6 & \\
\hline 31 & $3 \mathrm{H}-5,90-105$ & 56 & Varve boundaries unclear & & & 1.6 & \\
\hline 31 & $3 \mathrm{H}-5,90-105$ & 57 & Varve boundaries unclear & & & 1.2 & \\
\hline 31 & $3 \mathrm{H}-5,90-105$ & 58 & Varve boundaries unclear & & & 2.0 & \\
\hline 31 & $3 \mathrm{H}-5,90-105$ & 59 & Nonlaminated sequence & 2.9 & 4.8 & & 3.9 \\
\hline 31 & $3 \mathrm{H}-5,90-105$ & 60 & Partial varve, not continuous & 1.6 & & & \\
\hline 31 & $3 \mathrm{H}-5,90-105$ & 61 & Varve boundaries unclear & 1.8 & 1.3 & & 1.6 \\
\hline 31 & $3 \mathrm{H}-5,90-105$ & 62 & Varve boundaries unclear & 1.6 & 2.2 & & 1.9 \\
\hline 31 & $3 \mathrm{H}-5,90-105$ & 63 & Varve boundaries unclear & & & 1.6 & \\
\hline 31 & $3 \mathrm{H}-5,90-105$ & 64 & Varve boundaries unclear & & & 2.5 & \\
\hline 31 & $3 \mathrm{H}-5,90-105$ & 65 & Varve boundaries unclear & 2.2 & 1.7 & & 2.0 \\
\hline 31 & $3 \mathrm{H}-5,90-105$ & 66 & Varve boundaries unclear & & & 2.3 & \\
\hline 31 & $3 \mathrm{H}-5,90-105$ & 67 & Varve boundaries unclear & & & 2.0 & \\
\hline 31 & $3 \mathrm{H}-5,90-105$ & 68 & Varve boundaries unclear & & & 2.9 & \\
\hline $3 i$ & $3 \mathrm{H}-5,90-105$ & 69 & Nonlaminated sequence & 8.4 & 7.0 & & 7.7 \\
\hline
\end{tabular}

Notes: Numbers were assigned sequentially from bottom to top for each varve, and for each nonlaminated interval. The thickness of each varved and non-laminated unit is reported preferentially as a mean of two measurements taken near the left and right margins of an X-radiographic image. In some cases, the limited resolution of an X-radiograph image made it necessary to take a single measurement near the center. 